\title{
Early or late gestational exposure to maternal immune activation alters neurodevelopmental trajectories in mice: an integrated neuroimaging, behavioural, and transcriptional study
}

Elisa Guma 1,2, Pedro Bordignon 3,5, Gabriel A. Devenyi 2,4, Daniel Gallino 2, Chloe Anastassiadis 2,6, Vedrana Cvetkovska 5, Amadou Barry ${ }^{3,11}$, Emily Snook 2,7, Jurgen Germann 2,8, Celia M.T. Greenwood ${ }^{3,9,10,11}$, Bratislav Misic ${ }^{12}$, Rosemary C. Bagot ${ }^{3,5}$, M. Mallar Chakravarty $1,2,4,13$

${ }^{1}$ Integrated Program in Neuroscience, McGill University, Montreal, QC, Canada

${ }^{2}$ Computational Brain Imaging Lab, Cerebral Imaging Center, Douglas Mental Health University Institute, Montreal, QC, Canada

${ }^{3}$ Ludmer Center for Neuroinformatics and Mental Health, Montréal, QC, Canada

${ }^{4}$ Department of Psychiatry, McGill University, Montreal, QC, Canada

${ }^{5}$ Department of Psychology, McGill University, Montreal, QC, Canada

${ }^{6}$ Institute of Medical Science \& Collaborative Program in Neuroscience, University of

Toronto

${ }^{7}$ Faculty of Medicine, University of Toronto, Toronto, ON, Canada

8 University Health Network, Toronto, Canada

${ }^{9}$ Lady Davis Institute for Medical Research, Jewish General Hospital, McGill University, Montreal, QC, Canada

${ }^{10}$ Department of Oncology, McGill University, Montreal, QC, Canada

${ }^{11}$ Departments of Human Genetics and Epidemiology, Biostatistics and Occupational Health, McGill University, Montreal, QC, Canada

12 Montreal Neurological Institute, McGill University, Montreal, QC, Canada

${ }^{13}$ Department of Biological and Biomedical Engineering, McGill University, Montreal, QC, Canada 


\begin{abstract}
Prenatal maternal immune activation (MIA) is a risk factor for neurodevelopmental disorders. How gestational timing of MIA-exposure differentially impacts downstream development remains unclear. Here, we characterize neurodevelopmental trajectories of mice exposed to MIA induced by poly I:C either early (gestational day [GD]9) or late (GD17) in gestation using longitudinal structural magnetic resonance imaging from weaning to adulthood. Early MIA-exposure associated with accelerated brain volume increases in adolescence/early-adulthood that normalized in later adulthood, in regions including the striatum, hippocampus, and cingulate cortex. Similarly, alterations in anxiety, stereotypic, and sensorimotor gating behaviours observed in adolescence normalized in adulthood. In contrast, MIA-exposure in late gestation had less impact on anatomical and behavioural profiles. Using a multivariate technique to relate imaging and behavioural variables for the time of greatest alteration, i.e. adolescence/early adulthood, we demonstrate that variation in anxiety, social, and sensorimotor gating associates significantly with volume of regions including the dorsal and ventral hippocampus, and anterior cingulate cortex. Using RNA sequencing to explore the molecular underpinnings of region-specific alterations in early MIA-exposed mice in adolescence, we observed the most transcriptional changes in the dorsal hippocampus, with regulated genes enriched for fibroblast growth factor regulation, autistic behaviours, inflammatory pathways, and microRNA regulation. This indicates that MIA in early gestation perturbs brain development mechanisms implicated in neurodevelopmental disorders. Our findings demonstrate the inherent strength of an integrated hypothesis- and data-driven approach in linking brain-behavioural alterations to the transcriptome to understand how MIA confers risk for major mental illness.
\end{abstract}




\section{Introduction}

Prenatal brain development is a complex process orchestrated by interacting genetic, environmental, and immune factors. During this period offspring are highly vulnerable to a variety of risk factors for neurodevelopmental disorders that may only emerge later in childhood or adolescence (1-3). Epidemiological and preclinical evidence supports maternal infection as a risk factor for neurodevelopmental disorders such as autism spectrum disorder (ASD) and schizophrenia acting as a disease primer $(1,4-6)$.

The effects of maternal infection are most often attributed to the maternal immune activation (MIA) rather than the specific pathogen. The increase in maternal proinflammatory cytokines disrupts the delicate immune balance between maternal and fetal environments, altering developmental processes (7-9). Studying the impact of MIAexposure in animal model offspring has been critical to establishing causality between MIA during pregnancy and downstream neurodevelopmental disruptions $(6,10,11)$. For example, MIA in pregnancy induces enduring behavioural, neuroanatomical, and transcriptional alterations relevant to schizophrenia and ASD (11-16).

However, neurodevelopmental processes and maternal cytokine responsiveness vary across gestation. Thus, the gestational timing of MIA-exposure may influence the nature and severity of disruptions in offspring (13,17-19). Epidemiological studies suggest exposure in early gestation confers the greatest risk for offspring (20-23). Nonetheless, there is significant variation in the literature with respect to the effects of MIA-timing. Previous animal studies comparing MIA-exposure in early (gestational day [GD] 9, the end of the human first trimester) or late (GD 17, the end of the human second trimester) report diverging neuroanatomical and behavioural phenotypes in adult offspring (17,24-26).

The emergence of neuroanatomical and behavioural abnormalities in the context of neurodevelopmental disorders is a dynamic process best characterized longitudinally. This is a strategy that has long been championed as a means for examining how variation from a "normative" path may lead to disordered brain development, potentially giving rise to neuropsychiatric complications (16,27-31). The effects of MIA are complex and may differ between regions, so whole brain strategies prioritizing developmental trajectories are particularly relevant in furthering our understanding of MIA-impact and moving beyond the many previous studies that have focused on isolated brain regions or behaviours in adulthood $(16,25)$. Furthermore, given the current COVID-19 global crisis, gaining an understanding of the long term effects of MIA is critical, as the number of MIA-exposed offspring is expected to rise (32-34).

Here, we examine the impact of early (GD9) or late (GD17) prenatal MIA-exposure on developmental trajectories in mice using longitudinal whole-brain magnetic resonance 
imaging (MRI) and multi-behavioural characterization. We identify a window of deviation from normative trajectories, specifically the adolescent/early-adult period, and apply an integrative analysis at this timepoint to derive a pattern of linked brain-behaviour covariation to integrate anatomical changes in affected regions, including the hippocampus, anterior cingulate cortex, striatum, septal nucleus with affected behaviours, including anxiety, stereotypy, and sensorimotor gating. Greater behavioural impairments were associated with volumetric decreases in regions including the hippocampus, thalamus, and cerebellum, and larger volume in the cingulate cortex and striatum. We use transcriptional profiling to probe the molecular underpinnings of this emergent brainbehaviour pattern and find dysregulation of genes regulating fibroblast growth factor signaling, immune signaling and autistic behaviours in one of the most affected regions, the dorsal hippocampus. Using multiple dimensions of brain anatomy, behaviour and gene expression we construct a comprehensive understanding of how MIA-exposure, particularly in early gestation, shapes offspring neurodevelopment. Taken together, these findings provide key insights into how exposure to this risk factor in gestation may increase susceptibility for neurodevelopmental disorders later in the lifespan. 


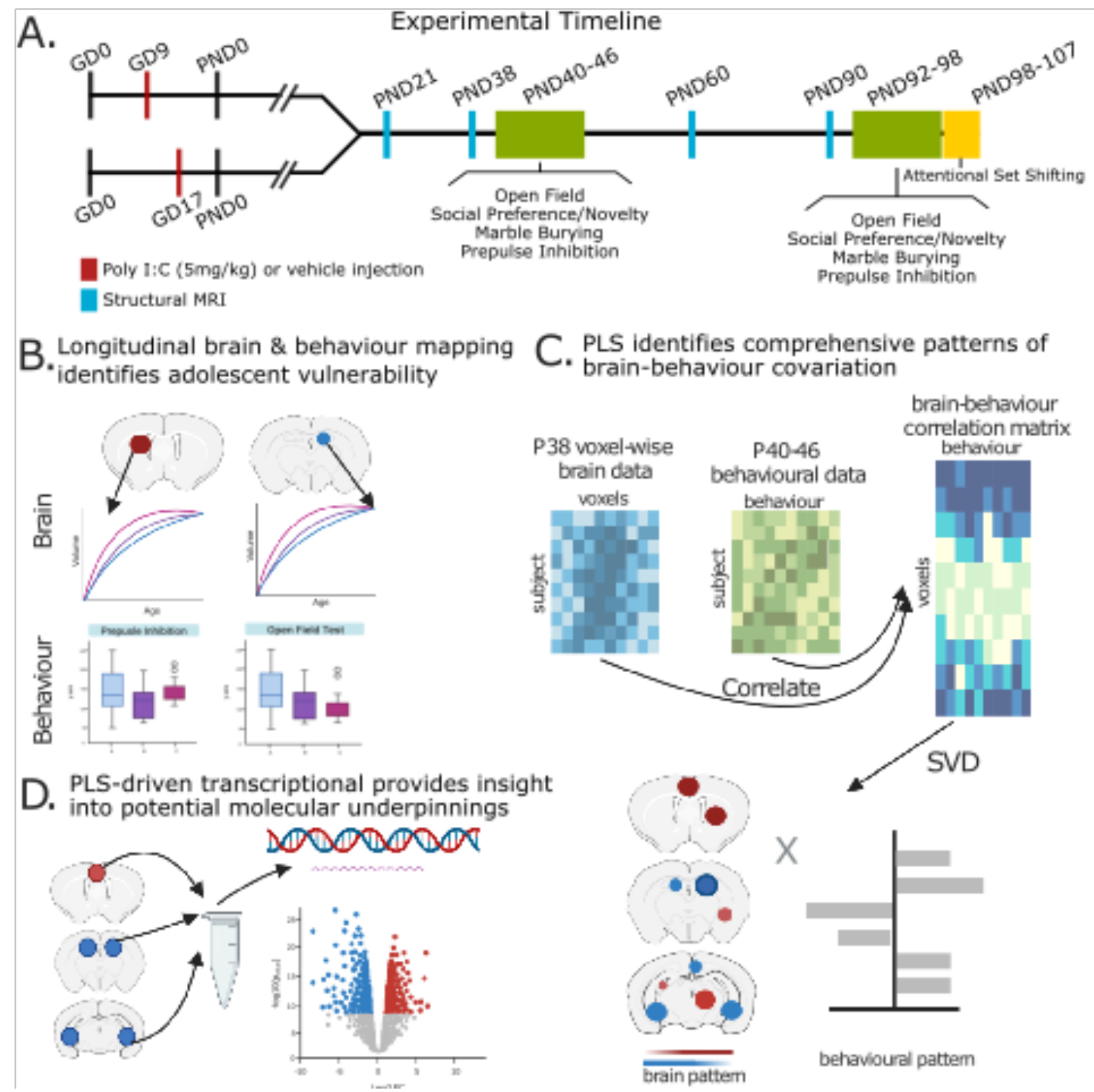

Figure 1. Experimental timeline. A. Pregnant dams were either injected (i.p.) with poly I:C $(5 \mathrm{~m} / \mathrm{kg})$ or vehicle ( $0.9 \%$ sterile $\mathrm{NaCl}$ solution) at gestational day (GD) 9 or 17 (red bars). Offspring were weaned and sexed on postnatal day (PND) 21. Longitudinal structural magnetic resonance imaging (MRI) was performed at PND 21, 38, 60, 90 (light blue bars). Two days following the PND38 (adolescence) and PND 90 (adulthood) scans, mice were assessed in open field test, social preference/novelty test ( 3 chambered social approach), marble burying task, and prepulse inhibition (green bars). The attentional set shifting task was also performed following the last adult behaviour (yellow bar). B. Univariate analyses were performed to assess group differences in neuroanatomy over time and behaviour for offspring exposed to MIA at GD 9, the early poly I:C (POL E) group, and offspring exposed at GD17, the late poly I:C group (POL L) group, relative to our combined vehicle exposed group, SAL (GD9 + 17). C. Partial least squares (PLS) was used to identify patterns of brain-behaviour covariation at the adolescent timepoint (PND38) where we 
observed our greatest group differences. This was used to identify regions of interest for RNA sequencing (D) used to probe potential molecular underpinnings of the observed changes. Figure made with BioRender https://biorender.com/.

\section{Results}

\subsection{Early and late gestational MIA-exposure differentially alter neurodevelopmental trajectory}

To test the effect of MIA-exposure at GD9 or 17 on brain development across the human equivalent of childhood (postnatal day [PND]21), adolescence (PND38), early adulthood (PND 60), and adulthood (PND90), we collected longitudinal in vivo structural magnetic resonance images $\left(\mathrm{MRI} ; 100 \mu \mathrm{m}^{3}\right)$ (Figure 1 ; section 4.2). We examined developmental trajectories using linear mixed-effects models at each voxel in the brain, testing for group by age (cubic natural spline fit) interaction, with sex as a covariate, and random effects for litter and mouse (section 4.2.3). This is akin to examining deviations from "normative" trajectories of brain development commonly performed in human neuroimaging studies $(27,35)$. We observed the most significant deviations in developmental trajectories in early exposed poly I:C (POL E) offspring relative to saline $(\mathrm{SAL})$ controls (for a quadratic age fit $\mathrm{t}=3.835,<1 \%$ false discovery rate [FDR]); POL $\mathrm{E}$ offspring had smaller brain volumes at P21, which then overshot between PND38 and PND60, and normalized at PND90. Many of these regions are implicated in neuropsychiatric and -developmental disorders such as the hippocampus, subiculum, cingulate cortex, striatum, nucleus accumbens, and septal nucleus (36-40) (Figure 2). Other important regions also affected include the periaqueductal gray and later developing regions such as the cerebellar vermis/crus I further detailed in supplementary figure 4. Offspring exposed to late poly I:C (POL L) had a flatter developmental growth trajectory relative to $S A L$ (for a cubic age fit $t=5.286,<1 \% F D R$ ), including the nucleus accumbens, auditory cortex, reticular nucleus, subiculum and hypothalamus, whereas the amygdala volume decreased in later adulthood (Figure 2 \& supplementary figure 4). These regions implicated in neuropsychiatric disorder show a somewhat different curve than that observed in the POL E group. POL E trajectories were significantly different from POL L, confirming that early MIA exposure had the largest effect on brain anatomy (section 4.2.3; supplementary materials 2.5 \& supplementary figures 9 \& 10). We also observed a significant monotonic increase in volume of cortical regions in POL E offspring (vs SAL) with a linear age fit; cubic age for POL E (vS SAL) and linear and quadratic age fits for POL L (vs SAL) were also significant, described in supplementary materials 2.3 \& 2.4 \& supplementary figures 5 -8. We also explored sex differences as a post-hoc analysis, as our model comparison did not suggest it was the optimal model for our data; 
POL E (vs POL L) males were more affected than females in a number of regions, but none were observed with SAL as the reference group (supplementary materials 2.6 \& supplementary figure 11).

A. POL E vs SAL * ns(age,2)
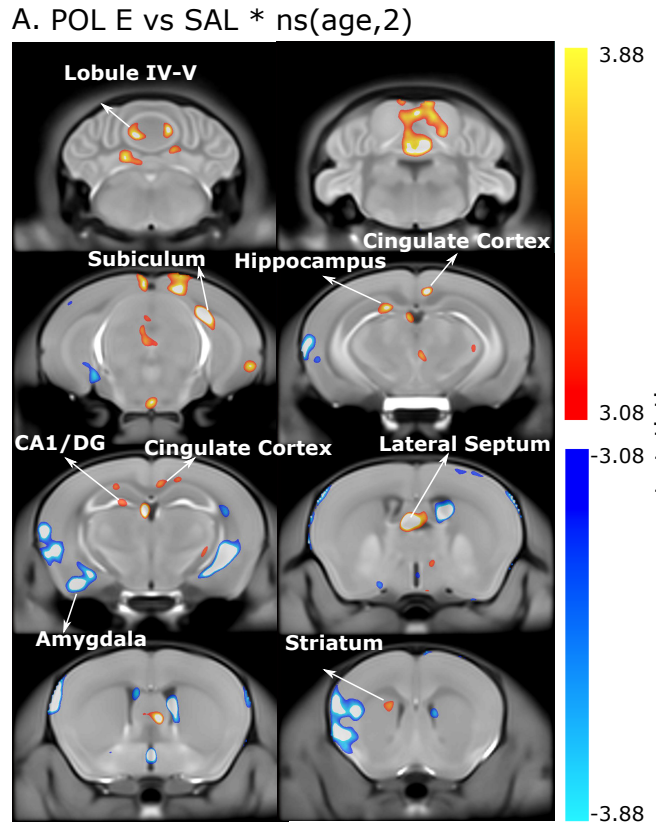

B.

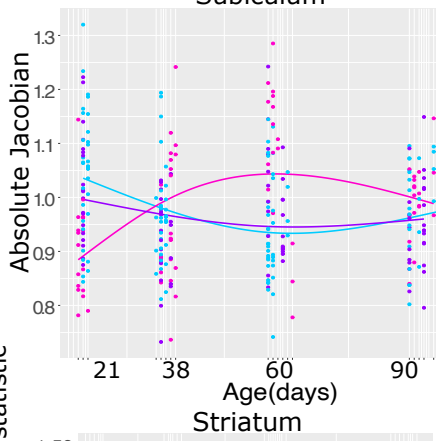

in 1.50

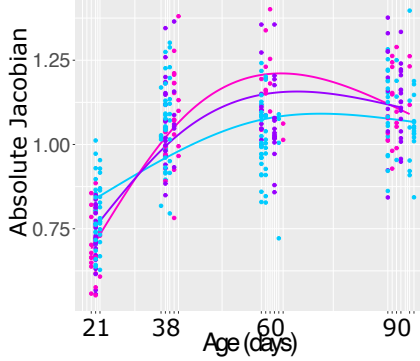

C. POL L vs SAL * ns(age, 3$)$

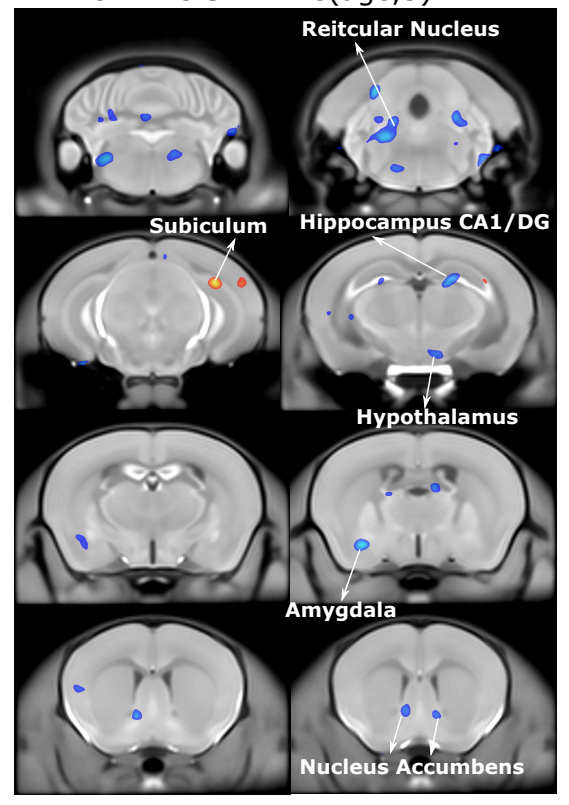

D.

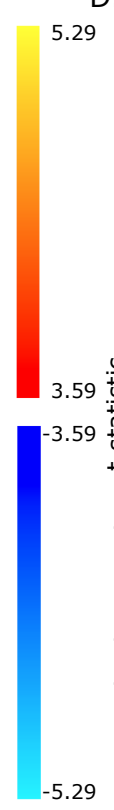

2.0. Hippcampus CA1/DG
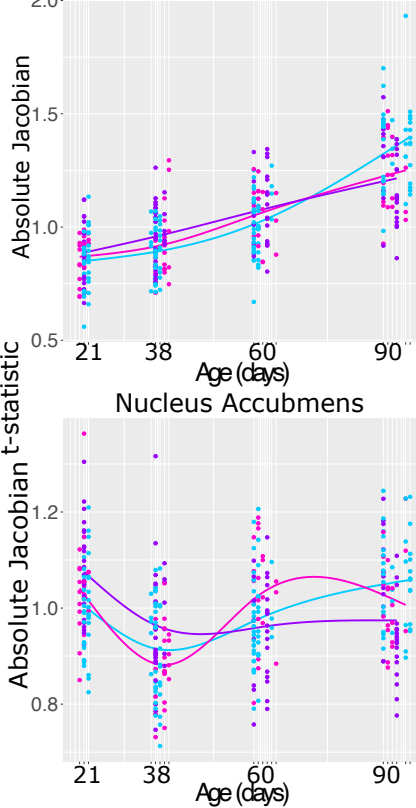

Nucleus Accubmens

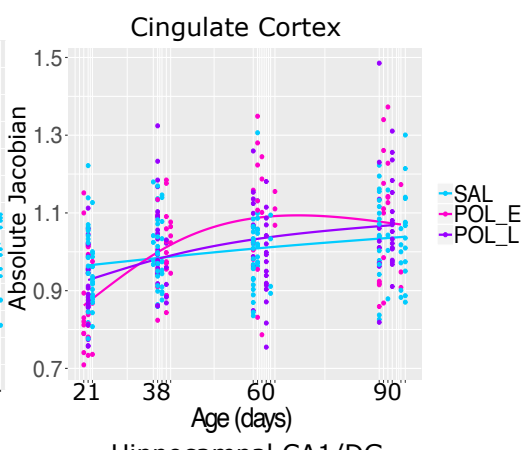

Hippocampal CA1/DG

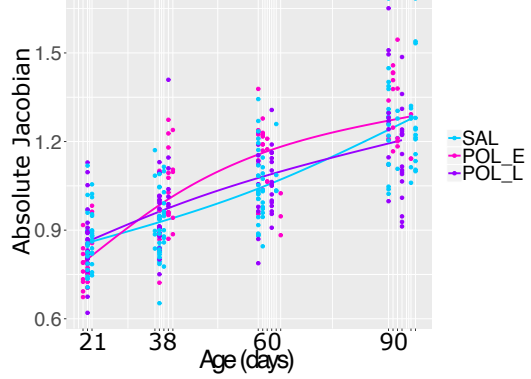

Hypothalamus
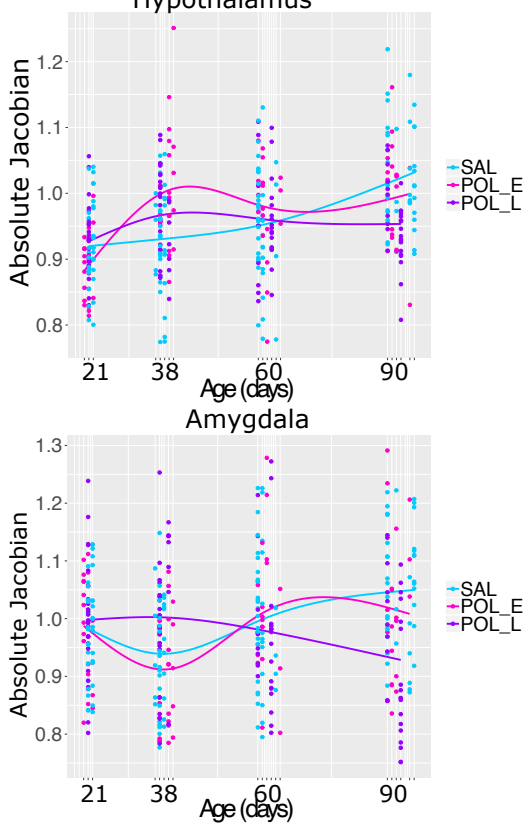

Figure 2. Developmental trajectories differ between early poly I:C group (POL E) vs saline controls (SAL) \& the late poly I:C group (POL L) vs SAL (thresholded at $5 \%$ False discovery rate (FDR)) (A) t-statistic map of group (POL E vs SAL) by age (quadratic natural spline) thresholded between 5\% FDR (bottom, $t=3.08$ ) and 1\% FDR (top, $t=3.83$ ) overlaid on the population (second level) average (B) Plot of peak voxels (voxel within a region of volume change showing largest 
effect) selected from regions of interest highlighted $(\mathbf{A})$, wherein age is plotted on the $x$-axis, and the absolute Jacobian determinants plotted on the $y$-axis. Here a value of 1 means the voxel is no different than the average, anything above 1 is relatively larger, and below 1 is relatively smaller. Ranges are not normalized to enhance comparison at each specific location in space. Trajectories are modeled as quadratic natural splines to reflect statistical modeling. (C) t-statistic map of group (POL L vs SAL) by age (cubic natural spline) thresholded between $5 \%$ FDR (bottom, $t=3.59$ ) and 1\% FDR (top, $t=5.29$ ). (D) Plots of peak voxels as described in (B) with curves modeled as cubic natural splines to reflect statistics.

\subsection{Early MIA-exposure induces behavioural alterations in adolescence}

To capture the developmental emergence of behavioural abnormalities, we performed a battery of tests to assay schizophrenia- and ASD- relevant behaviours in the same animals that underwent neuroimaging (section 2.1) after the adolescent (PND38) and adult (PND90) scans. We assessed exploratory behaviour and anxiety (open field test), social behaviour (three chambered social preference task), stereotypy (marble burying task), and sensorimotor gating (prepulse inhibition to acoustic startle) (Figure 1; section 4.3).

In adolescence, POL E offspring traveled less than SAL in the anxiogenic center zone of the open field relative to total distance of the arena, however this effect did not survive multiple comparisons correction (uncorrected $p$ values reported, and Bonferroni $q$ values, $a=0.05 / 11=0.0045 ; \quad t=-2.294$, uncorrected $p=0.039$, $q=0.429$; section 4.3.2; Figure 3A). They buried more marbles than $S A L$ in the marble burying task, potentially suggesting greater stereotypy/anxiety $(t=2.937, p=0.003, q=0.033$; Figure $3 B$ ). Relative to SAL, POL E displayed a striking impairment in sensorimotor gating $(t=-4.202, p=4.0 \times$ $10^{-7}, \mathrm{q}=4.0 \times 10^{-6}$; Figure $3 \mathrm{C}$ ) across prepulse tones (i.e. no interaction between group and prepulse level: $t=-0.995, p=0.321$; Figure 3D). Surprisingly, adolescent offspring showed no impairments in social preference or novelty Figure 3E). Moreover, behavioural alterations were no longer present in POL E offspring in adulthood apart from a subthreshold impairment in social novelty behaviour ( $t=-2.369, p=0.0311, q=0.341$; Figure 3F), wherein POL E mice spent more time exploring the familiar than the novel social target.

Adolescent POL L offspring did not show any significant behavioural alterations. Some subtle behavioural alterations emerged in adulthood, however they did not survive Bonferroni correction. Details in supplementary section 2.7 and results summary in supplementary table 3. Only subthreshold effects were observed in the attentional set shifting task (ASST) performed only in adulthood, (supplementary materials 2.7.1 \& supplementary figure 12). Post-hoc analyses of sex-differences are reported in supplementary materials 2.7.2 \& supplementary figure 13. 
A.

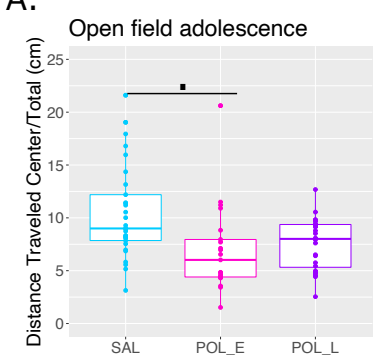

C.

Prepulse inhibition adolescenc

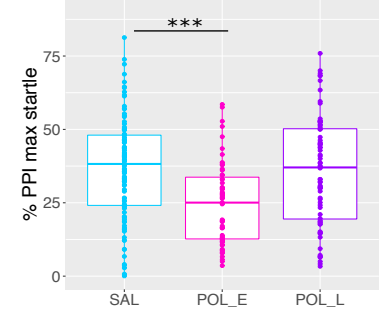

E. Social preference adolescence

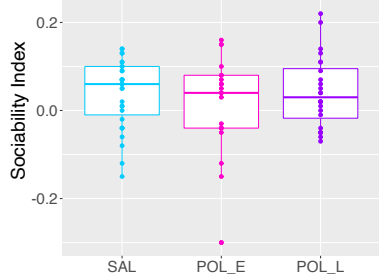

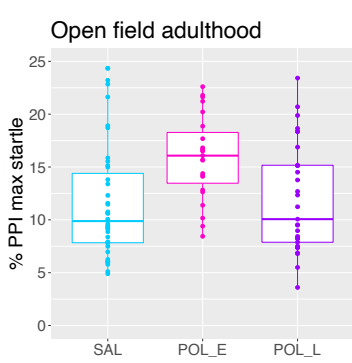

Prepulse inhibition adulthood

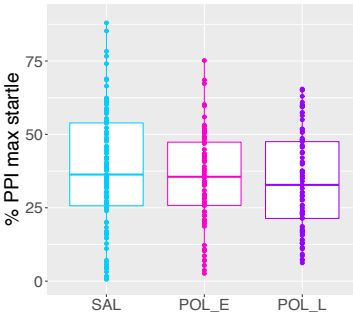

Social preference adulthood
B.

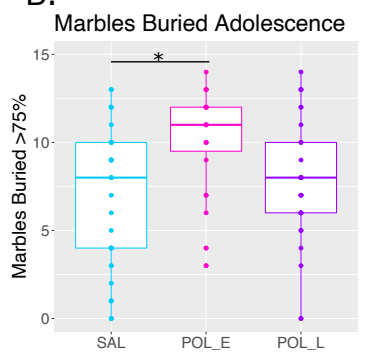

D.

Prepulse inhibition adolescencє

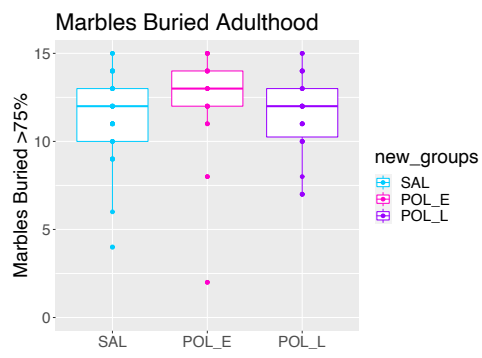

Prepulse inhibition adulthood
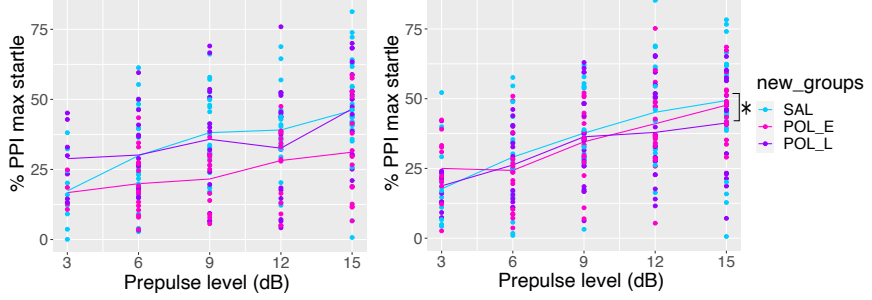

F.Social novelty adolescence

Social novelty adulthood
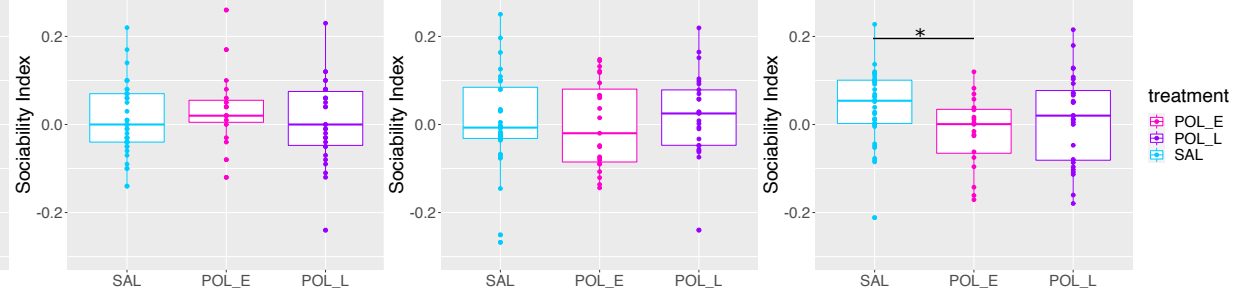

Figure 3. Early MIA-exposure induces transient behavioural impairments whereas late MIAexposure does not affect behaviour. Behavioural results for adolescent (left) and adult (right) offspring from the three treatment groups: SAL (cyan), POL E (magenta), POL L (purple). For all boxplots the midline represents the median of the data, the box represents the interquartile range, with whiskers denoting the full range of the data. (A) In adolescence (left), POL E offspring travel less in the center zone (relative to the total distance traveled) at a subthreshold level $(\mathrm{t}=-2.294$, $\mathrm{p}=0.039$ not significant following Bonferroni correction). No statistically significant differences observed in adulthood (right). (B) Significantly more marbles are buried by POL E adolescent offspring (left; $t=2.937, p=0.003$ ) than $S A L$ offspring. No group differences were observed in adulthood (right). (C) POL E offspring show a significant decrease in \% prepulse inhibition based on maximum amplitude of startle reaction to startle tone (left; $t=-4.202,4.0 \times 10^{-7}$ ). This deficit is no longer present in adulthood (right). (D) \%PPI is plotted for each group with increasing prepulse tone on the $x$-axis and \%PPI based on maximum amplitude of startle reaction on the $y$-axis for adolescence (left) and adulthood (right). No significant differences in slopes are observed between groups, however the POL E offspring are impaired at all levels in adolescence. (E) No significant differences in sociability index for the social preference task (i.e. preference for novel mouse over nonsocial object) between groups at either adolescence (left) or adulthood (right). (F) No significant differences in sociability index for social novelty (i.e. preference for novel mouse 
over familiar mouse) between groups. A subthreshold impairment was observed in POL E adult

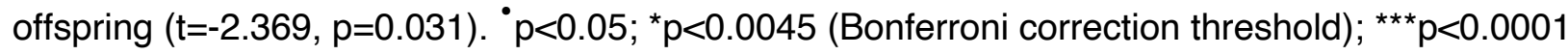

\subsection{Multivariate analysis of brain-behaviour data links variation in autism- and schizophrenia-related behaviours to volume changes in key brain regions}

Based on the analyses described in sections 2.1 \& 2.2 we identified the adolescent/early adult period as one of greatest deviation from "normative" trajectories (particularly for the POL E group). Focusing on single modalities (neuroimaging or behaviour only) may obscure more complex relationships between brain and behaviour. To probe these potentially critical associations, we employed a data-driven technique, partial least squares (PLS), to perform multivariate mapping between whole-brain anatomical alterations and adolescent behavioural metrics across 4 different tests (PND 38; Figure 1; section 4.4). This analysis reveals patterns of covariation that link patterns of disordered brain development with disordered behaviours by identifying 'latent variables'.

We identified two significant latent variables (LVs); LV1 described a pattern of brain-behaviour covariation (29\% covariance explained, $p=0.034)$, whereas LV2 described a brain pattern associated with sex and litter size (19\% covariance explained, $p=0.002$ ) (Figure 4A; supplementary materials 2.8 \& supplementary figure 14). For this reason, we chose to focus on LV1. We observed a pattern of attenuated behavioural impairment, i.e. decreased locomotion and anxiety (open field test), more social interactions (social preference and novelty), and less impairment in sensorimotor gating (PPI) that was associated with larger volume of the ACC, somatomotor cortex, and striatum. A pattern of greater behavioural impairment, including increased anxiety and locomotion, fewer social interactions, and impaired sensorimotor gating was associated with smaller volume in the dorsal and ventral hippocampus, thalamic nuclei, and cerebellum (vermis, crus I and II) (Figure 4 B\&C).

Inspecting correlations between the brain-behaviour weights for each mouse, colored by group, suggests that the POL E offspring load more strongly on this pattern than the other two groups, albeit not for all subjects (Figure 4D; supplementary figure 15 shows the brain-behaviour correlation plot after removal of an outlier on behavioural loadings circled on plot). Finally, to determine how this pattern changes in adulthood, we applied brain-behaviour weights for LV1 computed in adolescence to the brain and behaviour data collected in adulthood. We observed a shift along the brain axis but not the behaviour axis, suggesting that, as mice age, changes in brain patterns are disconnected from changes in behavioural patterns (supplementary figure 16). 

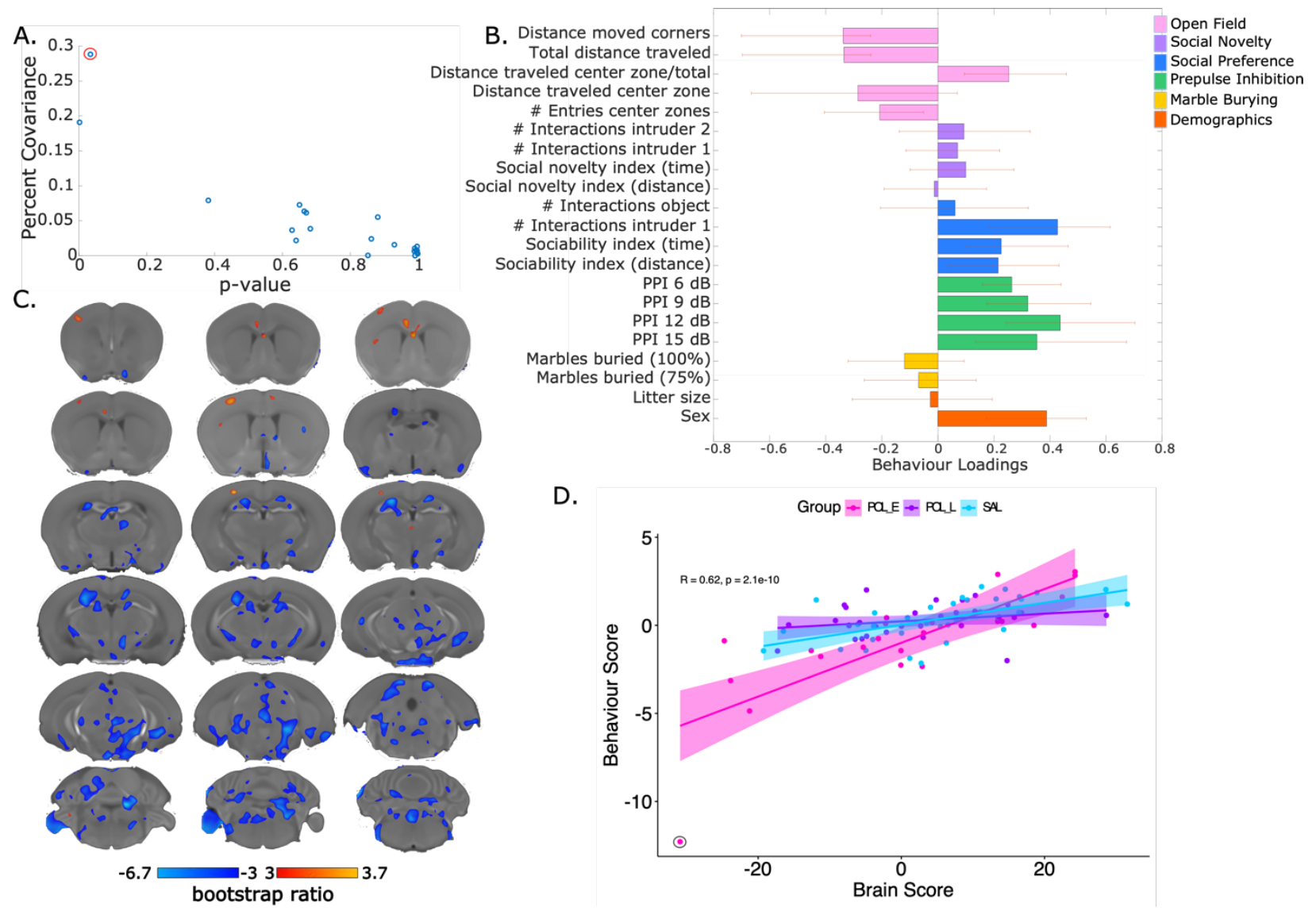

D.

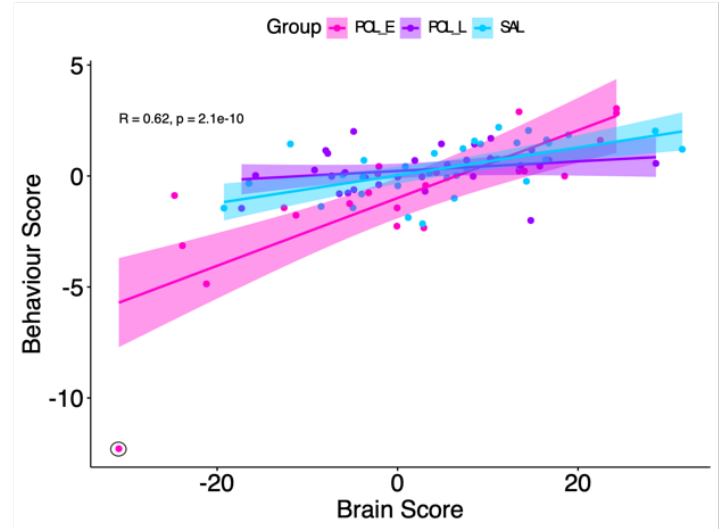

Figure 4. Partial least squares (PLS) analysis results for first latent variable (LV1). (A) Covariance explained (y-axis) and permutation p-values (x-axis) for all 21 LVs in the PLS analysis. LV1 is circled in red $(p=0.034$, \%covariance $=29 \%)$ and was chosen for subsequent investigation based on the covariance explained and behavioural relevance of results. (B) Behaviour weight for each behavioural measure included in the analysis showing how much they contribute to the pattern of LV1. Singular value decomposition estimates the size of the bars whereas confidence intervals are estimated by bootstrapping. Bars with error bars that cross the 0 line should not be considered. (C) Brain loading bootstrap ratios for the LV1 deformation pattern overlaid on the population average, with positive bootstrap ratios in orange-yellow (indicative or larger volume), and negative in blue (indicative of smaller volume). Colored voxels make significant contributions to LV1. (D) Correlation of individual mouse brain and behaviour score, color coded by treatment group with a trend line per group. Outlier on the behaviour score circled in dark gray. Early poly $\mathrm{I}: \mathrm{C}$ (POL E) offspring (magenta) express this pattern more strongly than the saline controls (SAL) and late poly I:C (POL L) groups. 


\subsection{Early MIA-exposure induces transcriptional changes in adolescence}

Based on our data-informed multivariate maps of brain-behaviour covariation, as well as a priori knowledge of regions implicated in schizophrenia- and ASD-related pathology $(38,41-47)$, we identified regions in which to probe underlying transcriptional alterations, namely the ACC, dHIP, and VHIP. We first profiled patterns of differential gene expression in adolescent POL E (vs SAL E) mice (Figure 1; section 4.5.2). Pooling all ROIs, we identified 962 genes significantly $(q<0.05)$ down-regulated and 668 genes upregulated in POL $E$ relative to SAL mice. We observed many differentially expressed genes (DEGs; $p<0.05$ ) in the dHIP ( 246 down- and 131 upregulated, $q<0.05$ ), with more subtle changes in the VHIP (37 down-, 12 upregulated, $q<0.05$ ) and ACC (17 down-, 0 upregulated, $q<0.05$ ) (Figure $5 \mathbf{A}-\mathbf{C}$ ). Several genes were significantly downregulated across all three ROIs, including Nfkbia, a key driver of the pro-inflammatory immune response for increasing cytokines production (48), as well as KIf2, Ddit4, and Per1 (Figure 5D \& E). Full gene lists are available in supplementary table 4.

Pathway enrichment analysis of genes upregulated in dHIP (section 4.5.3) identified enrichment of fibroblast growth factor signaling (including FGF1, 2 and 3), an evolutionarily controlled signaling pathway critically involved in embryogenesis and synaptogenesis (49). Autistic behaviour and regulation of microRNAs (including miR466i-3p, miR-362-3p, miR-329-3p) were also enriched. Downregulated dHIP genes were enriched for apoptosis, a critical neurodevelopmental process previously shown to be disrupted by MIA-exposure $(50,51)$ and microRNAs (including miR-3097-3p, miR-499-5p, miR-717). Enrichment of NF-kappa-B inhibitor alpha signaling and erythrocyte differentiation enrichment, both implicated in the immune system, was also observed $(52,53)$ (Figure 5E; supplementary table 5).

In the vHIP, downregulated genes were enriched for IL-17 signaling pathway, previously shown to be critical for promoting an ASD-like phenotype in MIA-exposed mice (54), apoptosis, NF-kappa-B inhibitor alpha signaling, chronic myeloid leukemia and small cell lung cancer.

ACC upregulated DEGs were enriched for white fat cell differentiation and IL-1 signaling, also involved in pro-inflammatory signaling (55), based on wikipathways (https://www.wikipathways.org/index.php/WikiPathways). We investigated sex differences but no striking differences were observed (supplementary materials 2.9.1).

We compared identified DEGs to those previously identified by transcriptional profiling of post-mortem human ASD (prefrontal and temporal cortex (56)) and schizophrenia samples (pan-cortical (56), and prefrontal cortex and hippocampus (57)). Although disease gene lists were not significantly enriched in the mice, FGF2 (significantly enriched in upregulated dHIP genes), Tbx4, and $C c d c 92$ were upregulated in both the dHIP of our mice, and the hippocampus of individuals with schizophrenia (57). 
Further, there was some overlap in downregulated genes across all 3 ROls and the pancortical SCZ sample, including Nfrkb (related to Nfkbia which was downregulated in all 3 ROIs), amongst others listed in supplementary table 6 . This further supports the relevance of our findings to neuropsychiatric disorders.

To explore the transcriptional synchrony between brain regions following prenatal MIA exposure, we used RRHO to identify genome-wide (threshold-free) patterns and significance of overlap between gene expression profiles across pairs of brain regions. We observed the strongest overlap in genes downregulated in both the dHIP and VHIP (4932) (supplementary figure 17A), however, there was a degree of overlap between all pairwise comparisons. Coordinately upregulated genes across brain regions were enriched for myelin associated processes, oxidative phosphorylation, and mitochondrial function. Genes coordinately downregulated across all ROls were enriched for RNA processing and transcriptional regulation. We report sex differences in gene overlap in supplementary materials 2.9 .2 \& supplementary figures 17B.

A.
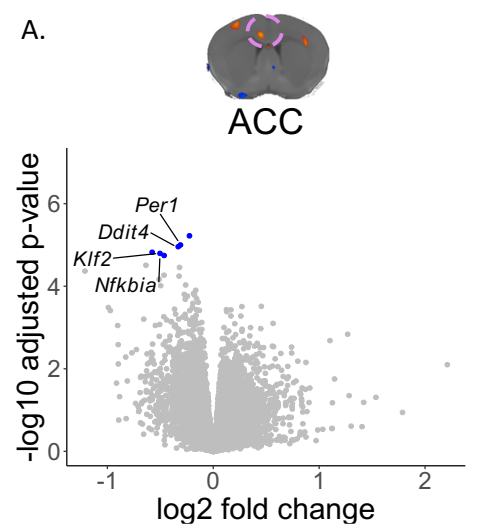

D. Stac3
Taf1c
B.
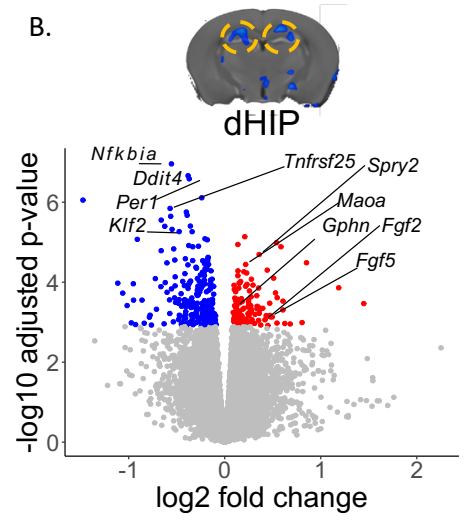

C.
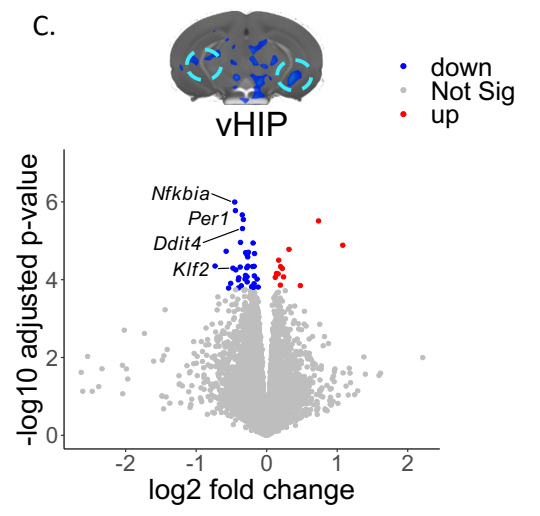

Downregulated

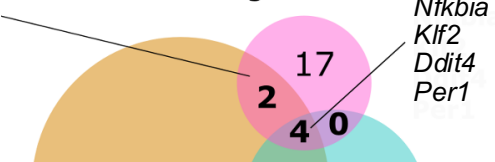

243

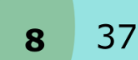

E. Upregulated

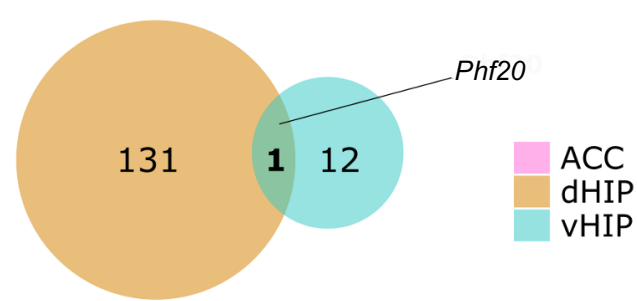


F. Regulation of FGF signaling

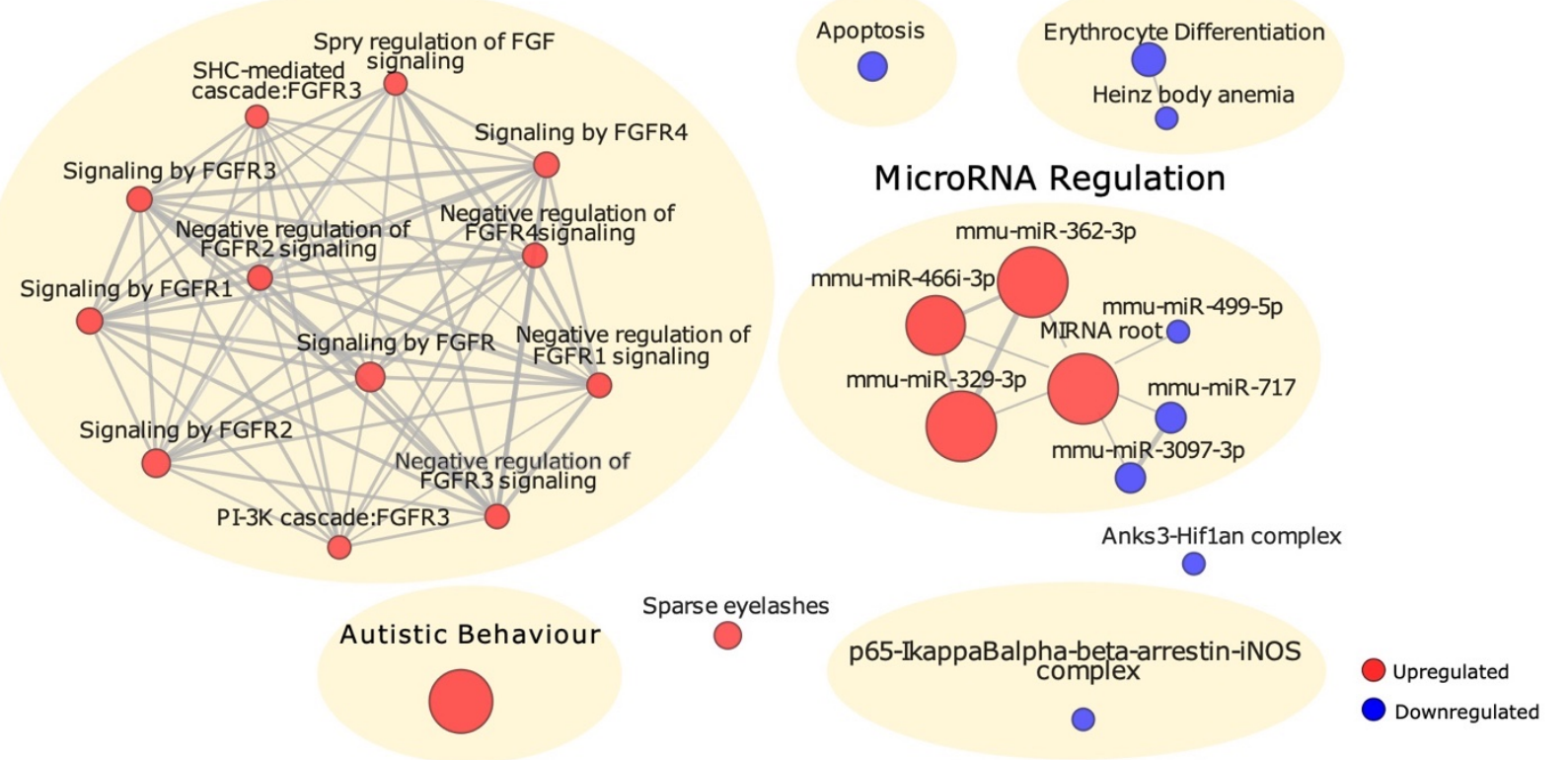

Figure 5. Transcriptional alteration in the adolescent brain at PND38 following MIA-exposure at GD9. Volcano plots for the ACC (A), dHIP (B), and vHIP (C) with significantly downregulated genes in blue, and upregulated genes in red. Genes that are either down- or upregulated in multiple ROIs (as shown in D \& E) are highlighted in the volcano plots (on the left side). For the dHIP volcano plot (B) gene names on the right hand side are enriched for FGF signaling, and also identified in human postmortem hippocampal samples from individuals with schizophrenia. Venn diagram showing overlap in downregulated (D) and upregulated (E) genes per ROI. F. Gene enrichment analysis results for the dHIP, with upregulated enrichment in red and downregulated in blue. Upregulated genes were significantly enriched for FGF signaling, as well as autistic behaviours, sparse eyelashes, and microRNA regulation. MicroRNA regulation was also enriched for downregulated genes, as were apoptosis, erythrocyte differentiation, heinz body anemia, and p65-IK $\beta K a-\beta$-arrestin-iNOS-complex. 


\section{Discussion}

In this study, we demonstrate that integrating brain and behavioural phenotypes can uncover key relationships between aberrant development due to MIA-exposure and the putative transcriptional underpinnings. Specifically, we present whole-brain longitudinal neuroimaging and multi-behavioural phenotyping of mice prenatally exposed to MIA at two different gestational timepoints to evaluate developmental trajectories from childhood to adulthood, finding that early MIA induces the most robust changes. Integrating data from the developmental window of greatest vulnerability to characterize patterns of brain-behaviour covariation by multivariate analysis, we identified key brain regions and investigated transcriptional alterations in adolescence in the most affected group, POL E, revealing putative molecular underpinnings of the observed MIA-induced brain-behaviour patterns. Based on these analyses, we conclude that prenatal MIAexposure early in gestation opens a window of vulnerability in adolescence/early adulthood characterized by an accelerated increase in brain volume and emergence of sensorimotor gating and stereotypy/anxiety-like deficits, both of which normalize in adulthood. These deviations may be driven by transcriptional changes in genes involved in FGF signaling, autistic behaviours, inflammatory pathways, and microRNA regulation particularly in the adolescent dHIP.

\subsection{The case for longitudinal investigation}

An accurate assessment of normal and abnormal brain development can be best achieved with a longitudinal approach. Our understanding of the emergence of neuropsychiatric disorders such as schizophrenia or ASD has been enhanced by longitudinal neuroimaging studies showing that deviations in brain development begin prior to disease onset $(58,59)$. Additionally, longitudinal studies are more sensitive, requiring fewer participants than cross-sectional studies to detect subtle differences in brain structure (60) and better account for interindividual differences (61). Volumetric changes ascertained with MRI provide a robust endophenotype in animal models that facilitate comparison to human illness $(62,63)$. Previous animal imaging studies from our own group have demonstrated the utility of a longitudinal design in the context of ageing, genotype associations, and treatments (64-68), while others provide important insight into early brain development (69) or sex differences in the developing brain (28).

Using our longitudinal approach we identified transient deviations in development due to MIA-exposure in early gestation which may have been missed had we focused solely on adulthood. This may, in part, explain why some cross-sectional studies examining MIA-induced neuroanatomical alterations have found differing results 
depending on age of testing (13). Future work should examine trajectories starting earlier and extending later in the lifespan.

\subsection{Early MIA-exposure is associated with greater deviations in neurodevelopmental trajectories}

MIA-exposure affected several regions often implicated in ASD or schizophrenia including the striatum, hippocampus and subiculum $(36-38,70)$, amygdala $(36,71)$, periaqueductal gray (72-74) (amongst others such as the somatosensory cortices (7577 ) and septal nuclei $(78,79)$ ). The shape of the developmental trajectories is also of significant interest. The overshoot in adolescence and early adulthood is reminiscent of the brain or cortical overgrowth phenomenon observed in ASD $(39,47,80,81)(39)$. Conversely, the altered hippocampal and ACC morphology is in line with psychosis spectrum disorders $(45,46)$. Previous MRI-based studies examining GD9 MIA-exposure have identified microstructural alterations in similar regions such as the ACC, hippocampus, lateral septum, and ventral striatum, albeit later in the lifespan $(25,26)$.

POL E offspring exhibited impairments in behaviours relevant to both ASD and schizophrenia, namely sensorimotor gating, stereotypy/repetitive behaviours, and subthreshold deficits in anxiety-like behaviours, consistent with previous reports of greater suppression of exploratory behaviours and sensorimotor gating impairments (17). However, we observed transient impairments in adolescence that were resolved in adulthood, whereas others have observed emergence of these deficits in adulthood, not adolescence $(16,17)$. This may be due to a number of factors including the dose of immunogen, route of administration, mouse strain, and more (82).

Exposure late in gestation led to subtler alterations in brain morphology, with some regions such as the amygdala showing decreases in adulthood, not paired with behavioural impairments. Previous cross-sectional MRI studies examining the impact of GD17 MIA-exposure in adult offspring report white matter and cerebellar volume decreases, and enlarged 4th ventricles $(25,83)$. Previous longitudinal studies examining MIA-exposure in mid-gestation (GD15) have identified enlarged lateral ventricles, often observed in MRI investigation of schizophrenia (36), as well as decreased cortical and hippocampal volume and altered microstructure all emerging in adulthood, providing further evidence for the impact MIA-timing on offspring outcomes $(16,25,26,84)$. Behaviourally, memory and cognitive deficits have been observed in GD17 MIA-exposed adult offspring $(17,24,85)$, whereas we only observed subthreshold deficits in cognitive performance in the ASST task. Importantly, previous work comparing the effects of GD9 and $17 \mathrm{MIA}$-exposure also observed diverging effects on offspring brain development $(17,25,86)$. Taken together, these findings indicate increases in maternal cytokine levels due to MIA that occur early in gestation may have more profound effects on offspring development . However, they also support the theory that MIA is a disease primer, as 
exposure to this risk factor alone does not seem to be sufficient in inducing long-term deficits later in the lifespan $(5,11,87,88)$.

Although speculative, it is worth considering what neurodevelopmental processes may be altered by the MIA-exposure at the gestational epochs we chose. At GD9 the developing brain is colonized by microglia, and neuronal and immune cell migration, neurogenesis, and cortical plate formation are being initiated $(3,89,90)$. At GD17 the organization of cortical layers and the hippocampus are underway, as are synaptogenesis, gliogenesis, and apoptosis $(1,5,91)$. It remains unclear to what extent MIA-exposure during these periods influences downstream neurodevelopmental changes across the differing scales of brain architecture. Previously, MIA exposure at GD9 has been associated with an increased density of activated microglia both in the embryo and adolescent brain; this may in turn interfere with synaptic pruning and circuit formation leading to aberrant neurodevelopment (92-94). Alterations to myelin-related structure and gene expression have been observed in GD17 MIA-exposed offspring (83). Finally, MIA in mid-gestation (GD12-15) has been associated with aberrant neurogenesis, reductions in the number of cortical neural stem cells, and reduced dendritic spine density $(95,96)$. Future work examining MIA at different gestational times (i.e. mid-gestation) with the integrative approach we developed may further our understanding of how MIA disrupts brain development.

\subsection{Identifying brain-behaviour associations}

One major limitation when examining neurodevelopmental phenotypes in either human or animal models is the tendency to assign phenotypes at the level of a single structure, or to examine associations between these structures and a specific symptom

or behaviour (97). Indeed, these strategies disregard the ever-increasingly acknowledged network-like architecture of the brain and the inherent relationships to behaviour $(98,99)$. In contrast, multivariate strategies, such as PLS, move beyond simplistic associations to better understand brain-behaviour relationships (100-102). In the context of small animal imaging, PLS provides a novel, streamlined method to assess cross-sectional brain-behaviour patterns from large cohorts of deeply phenotyped mice. This analysis, seldom applied to preclinical studies, has been widely applied to human neuroimaging studies investigating, for example, the relationship between neuroanatomy and gene expression (103) or clinical and cognitive symptoms in schizophrenia (104). This integrative analysis permits inclusion of multiple measures from the same individual while accounting for their potential inter-relatedness. Further, it balances hypothesis driven experimental choices (brain regions, behaviours tested, etc) with hypothesis-free data-driven investigation of their relationships. Finally, it may promote cross-species 
translation by improving methodological homology between human and rodent neuroimaging studies.

\subsection{Neuroimaging-driven RNA sequencing reveals potential molecular underpinnings of brain-behaviour relationships.}

We observed significant enrichment of gene ontology terms for FGF signaling pathways, as well as autistic behaviour, and microRNA regulation amongst upregulated dHIP genes. Interestingly, we also saw enrichment of immune pathways such as IL-17 in the vHIP, IL-1 in the ACC, and NFK-B in the d- and VHIP in line with previous findings (54). This provides further evidence for immune system dysregulation following MIAexposure, which may interfere with neurodevelopmental processes such as neuronal migration, microglial function, and immune system development $(8,105,106)$.

FGFs are signaling proteins that influence the development and repair of most mammalian tissues $(107,108)$. They play a critical role in embryonic development, influencing the growth and patterning of several brain structures, cell survival and proliferation, neurogenesis and neuronal repair $(109,110)$. In preclinical studies, FGF signaling inhibition has been observed to disrupt cortical gyrification, neural progenitor development in the subventricular zone of the developing cerebral cortex (111), hippocampal excitatory and inhibitory synaptogenesis and cell maturation $(112,113)$, and left-right symmetry, all phenomena observed in schizophrenia and autism (114). Enhanced FGF signaling has been associated with beneficial effects, such as reduced anxiety, enhanced neurogenesis, and reverses hippocampal and cortical atrophy $(115,116)$. Dysregulation of FGF signaling has been proposed to increase vulnerability to ASD, producing variation in brain growth and cortical circuit formation $(117,118)$. Further, a few of the FGF signaling genes we observed to be regulated have also been implicated in schizophrenia in human post-mortem brains (57). FGF receptor 2 single-nucleotide polymorphisms associate with schizophrenia $(108,119,120)$; this gene was significantly upregulated both in the dHIP of our early MIA-exposed mice and the hippocampi of individuals with schizophrenia (57).

\subsection{Limitations}

The results and discussion of this paper should be considered in light of their limitations. No animal model can fully recapitulate the human condition, therefore not all observations made here may apply to human pathology and brain development (89). However, we do observe interesting parallels between our MIA-exposed mice and psychiatric patients' altered trajectories of brain development, behaviour, and transcription. Further, studies investigating the impacts of MIA exposure in humans also report that earlier exposure induces more serious downstream effects on offspring (see 
(13) for a review). Although both the neuroimaging and behavioural studies conducted were longitudinal, our multivariate and transcriptional analyses were cross-sectional. Future work should be done to extend these analyses across the lifespan. We investigated sex differences; however we did not detect statistically significant interactions in neuroanatomy or behaviour. Although we collected data for both male and female offspring, we may still have been underpowered to detect potentially subtle sexspecific effects. When comparing our two POL groups directly, we did observe greater alterations in male POL E offspring, which may indicate existence of subtle sex-effects $(121,122)$. Surprisingly we did not observe any strong social deficits, often reported in MIA-offspring and central to ASD pathology $(11,54,123)$. This may be a function of MIAtiming, age of investigation, dose of immunogen used, or route of administration. In contrast to our findings, previous studies observed MIA-offspring deficits in adulthood $(16,17)$; it is possible that these may emerge later in the lifespan or following exposure to an additional risk factor.

\subsection{Conclusions}

We comprehensively examined the effects of prenatal MIA-exposure, a known risk factor for neuropsychiatric disorders, at two different gestational timepoints, on offspring brain and behaviour development using a robust translational measure: in vivo rodent imaging. We applied multivariate statistical analyses to integrate these modalities, leveraging this to investigate underlying transcriptional changes in the group and age at which we detected the greatest changes. Taken together, these findings suggest that prenatal MIA-exposure early in gestation may interfere with critical neurodevelopmental processes more so than exposure in late gestation. This leads to transient deviations in brain and behaviour development in adolescence and early adulthood, potentially increasing susceptibility to other risk factors, but, which, in the absence of subsequent challenge, normalize later in adulthood. These may be linked to altered transcription of genes involved in FGF signaling and inflammatory pathways. 


\section{Methods}

\subsection{Animals, Breeding \& Maternal Immune activation protocol}

Timed-mating procedures were used to generate pregnant dams, who were injected intraperitoneally with either poly I:C (POL; P1530-25MG polyinosinicpolycytidylic acid sodium salt TLR ligand tested; Sigma Aldrich) $(5 \mathrm{mg} / \mathrm{kg})$ or vehicle (SAL; $0.9 \%$ sterile $\mathrm{NaCl}$ solution) at GD 9 or 17 resulting in 4 groups: POL E (5 dams), SAL E (4 dams), POL L (6 dams), SAL L (5 dams). Experimental design is outlined in Figure 1 (see Supplementary materials $\mathbf{1 . 1}$ for breeding, model, birth and weaning details). We confirmed the immunostimulatory potential of our poly I:C in a separate set of dams (details for procedure in supplementary materials 1.6 and results in supplementary materials 2.1 \& supplementary table 2 ).

\subsection{Magnetic resonance imaging}

\subsubsection{Acquisition}

Longitudinal T1-weighted $\left(100 \mu \mathrm{m}^{3}\right)$ structural MRI scans were acquired in vivo at PND 21 ( childhood), 38 ( adolescence), 60 ( young adulthood), and 90 ( adulthood) $(89,90)$ at the Douglas Institute in MIA- or SAL-exposed offspring (7 Tesla Bruker Biospec 70/30; matrix size of $180 \times 160 \times 90 ; 14.5$ minutes, 2 averages) $(64-66,68) . \mathrm{MnCl}_{2}(62.5$ $\mathrm{mg} / \mathrm{kg}$ ) was used for contrast enhancement $(60,124)$, and isoflurane to anesthetize the mice (5\% induction, $1.5 \%$ maintenance) (Supplementary materials $\mathbf{1 . 3}$ for more details).

\subsubsection{Image processing}

Images $(n=376)$ were exported as Digital Imaging and Communications in Medicine (DICOM), converted to the Medical Imaging NetCDF (MINC) format, preprocessed to enable downstream analyses, and visually inspected for quality control (QC; $\quad \mathrm{n}=27$ scans were excluded https://github.com/CoBrALab/documentation/wiki/Mouse-QC-Manual-(Structural)). 
Preprocessed images were used as inputs for the two-level Pydpiper registration toolkit to perform longitudinal deformation based morphometry analysis (https://wiki.mouseimaging.ca/pages/viewpage.action?pageld=1868779) Briefly, in the first level, affine and non-linear registration was used to create a subjectaverage per mouse (by registering all scans for one mouse). In the second level all subject averages were registered to create a study average and a common space for statistical analysis (125) (Supplementary figure 1 for workflow). Quality control (QC) was performed through visual inspection to ensure that registrations worked as expected. Final numbers per group/timepoint are presented in table 1.

Table 1. Sample per timepoint following quality control. Postnatal day (PND); poly I:C (POL); saline (SAL); late (L; gestational day 17 exposure); early (E; gestational day 9 exposure); male $(\mathrm{M})$; female $(\mathrm{F})$.

\begin{tabular}{|lr|l|l|l|l|}
\hline & PND 21 scan & PND 38 scan & PND 60 scan & PND 90 scan \\
\hline $\begin{array}{l}\text { SAL E + L } \\
\text { litters) }\end{array}$ & $37(19 \mathrm{M} / 18 \mathrm{~F})$ & $38(20 \mathrm{M} / 18 \mathrm{~F})$ & $41(21 \mathrm{M} / 2 \mathrm{FF})$ & $40(20 \mathrm{M} / 20 \mathrm{~F})$ \\
\hline $\begin{array}{l}\text { POL E } \\
\text { litters) }\end{array}$ & $\mathbf{( 5}$ & $21(8 \mathrm{M} / 13 \mathrm{~F})$ & $21(9 \mathrm{M} / 12 \mathrm{~F})$ & $20(8 \mathrm{M} / 12 \mathrm{~F})$ & $20(8 \mathrm{M} / 12 \mathrm{~F})$ \\
\hline $\begin{array}{l}\text { POL L } \\
\text { litters) }\end{array}$ & $(\mathbf{6}$ & $27(12 \mathrm{M} / 15 \mathrm{~F})$ & $29(13 \mathrm{M} / 16 \mathrm{~F})$ & $27(14 \mathrm{M} / 13 \mathrm{~F})$ & $28(14 \mathrm{M} / 14 \mathrm{~F})$ \\
\hline
\end{tabular}

Both relative (nonlinear deformations with residual affine removed) and absolute (linear plus nonlinear deformations) Jacobian determinants (127) of the deformation fields of the first level were resampled into the second level final average space in order to perform statistics, and blurred with a $0.2 \mathrm{~mm}$ full-width-at-half-maximum 3D Gaussian to better conform to Gaussian assumptions for downstream statistical testing $(125,126)$. Further detail in supplementary materials 1.3 and supplementary figure 1.

\subsubsection{Statistical analysis}

Statistical analyses were performed using the $R$ software package ( $R$ version 3.5.1, RMINC version 1.5.2.2 www.r-project.org). Linear mixed-effects models (Ime4_1.121 package) were chosen for our longitudinal analyses as they are robust to missing data, and allow for fitting both fixed and random effects for the same subject over time. To select the most appropriate natural spline fit for our age term, we compared increasingly complex models with the log-likelihood ratio test at every voxel in the brain following 
similar methodology presented in previous studies (Supplementary materials 1.7.1) $(28,69)$. Modeling age with a cubic natural spline fit best explained the variance in our data, with group and sex as fixed effects, and litter and mouse id as random intercept effects (supplementary section 2.2 and supplementary figure 3 ). SAL E and $L$ offspring were merged into a single group after ensuring they were not significantly different (Supplementary materials 1.7.2). A voxel-wise linear mixed-effects model was applied to the first level absolute Jacobian determinant (to examine within-subject trajectories) to assess the effect of MIA-exposure on development with an age (cubic natural spline fit) by group interaction, covarying for sex (fixed effects), while subject and litter were modeled as random intercept effects. The model was run again with POL $L$ as the reference group in order to compare POL E and POL L directly. The False Discovery Rate (FDR) (128) correction for multiple comparison was applied to our statistical tests. As a follow up analysis, we investigated whether there was a sex by group by age (cubic nonlinear spline) interaction (supplementary materials 2.3).

\subsection{Behavioural testing}

\subsubsection{Behavioural protocols}

Tests were performed following the adolescent (PND38) and adult (PND90) scans, with a 2 day resting phase between tests and 1 hour habituation to the behavioural room prior to the test. Videos were analyzed using the Ethovision XT 12 tracking system (Noldus Information Technology, Leesburg, VA, USA).

Briefly, the open field test was used to assess exploratory behaviour and anxiety by comparing the distance traveled in the anxiogenic center zone $\left(40 \%\right.$ of a $45 \times 45 \mathrm{~cm}^{2}$ grey plastic arena) compared to the corners and edges. Mice were allowed to explore the arena for 15 minutes under bright light.

For the three chambered social approach task, mice were habituated (10 minutes) under red light to a three-chamber plastic box $(26(\mathrm{l}) \times 21.6(\mathrm{w}) \times 21.6(\mathrm{~h}) \mathrm{cm})$ with divider panels that have open doors, with a wire container $(9.5(\mathrm{~h}) 7.6(\mathrm{~d}) \mathrm{cm})$ in the two extreme chambers. To measure social preference (10 minutes), interactions with an age-matched same-sex stranger mouse were compared to interactions with a non social object (each under wire containers); to measure social novelty, interactions were compared between the same stranger mouse and a novel stranger mouse (which replaced the non social object).

The marble burying task was used to assess stereotypic and repetitive digging behaviour. We measured how many of 15 equidistantly spaced marbles mice buried in 
30 minutes under standard wood chip bedding ( $\sim 7 \mathrm{~cm}$ deep layer) in a standard home cage $(28(h) \times 17(w) \times 12.7(h) c m)$; see (129).

Sensorimotor gating to acoustic startle was measured with the prepulse inhibition task using commercially available startle chambers (San Diego Instruments, San Diego, CA). Startle response to an acoustic stimulus (120 dB) was compared to trials in which the startle stimulus was preceded by a $30 \mathrm{~ms}$ prepulse stimulus ranging from 3$15 \mathrm{~dB}$ above background noise (73-85 dB; 5 trials/stimulus, 3dB increments).

An additional cognitive flexibility and reversal learning measure (attentional set shifting task [ASST] (130)) was performed following PND90 behaviours to determine if there were lasting effects of the MIA-exposure, as previously reported in adult MIAexposed offspring in late gestation (17) (supplementary materials 1.4 for details). Mice were perfused following the last behaviour (supplementary materials 1.5).

\subsubsection{Statistical analysis}

We used linear mixed-effects models for adolescent and adult behavioural data, with group and sex (SAL and male as references) as fixed effects, and litter as a random effect to account for possible litter variability. A Bonferroni correction was applied ( 5 tests in adolescence, 6 in adulthood: $a=0.05 / 11=0.0045$ set as significance threshold, uncorrected $\mathrm{p}$-values, and corrected q-values reported). As with the neuroanatomy, a follow-up assessment of sex differences was carried out (supplementary materials 2.7.2.).

\subsection{Partial Least Squares Analysis}

Partial Least Squares (PLS) is a multivariate analysis which allows us to find the optimal weighted linear combination of two variables (voxel-wise DBM and behavioural metrics) that maximally covary together (131-133). A covariance matrix was computed from the z-scored brain (voxel-wise DBM measures) and behaviour (relevant behavioural metrics, PND38 tests, sex, and litter) matrices. Singular value decomposition was applied to the covariance matrix (134) to yield a set of orthogonal latent variables (LVs). This generates a set of 'brain weights' and 'behaviour weights' describing how each voxel or behavioural variable, respectively, weighs onto a given LV, and a singular value, describing the proportion of covariance explained by the LV. Brain and behaviour weights were projected onto individual subject data to generate subject-specific brain and behaviour scores. Permutation testing ( $n=1000$ repetitions) was used to assess the statistical significance of each LV and bootstrap resampling $(n=1000)$ was applied to assess the contribution of original brain and behaviour variables to each LV. Bootstrap 
ratios were thresholded at values corresponding to a $95 \%$ confidence interval $(131,135)$. Further details can be found in supplementary materials 1.7.3.

\subsection{Transcriptional analysis}

\subsubsection{Brain Extraction and RNA Isolation}

A separate cohort of mice (PND38; POL E $n=6 M / 6 F, 5$ litters, SAL E n=6M/6F, 6 litters) were euthanized in their home cage, brains were rapidly extracted and placed in chilled PBS and sliced (1 mm thick sections); tissue was punched from the ACC (bregma $+0.14 \mathrm{~mm}$ ), dHIP (bregma $-2.80 \mathrm{~mm}$ ), and VHIP (bregma $-3.08 \mathrm{~mm}$ ) and flash frozen on dry ice (death to freezing all samples $<3$ minutes). RNA extraction was performed using ReliaPrep Tissue RNA Miniprep system (Promega). RNA quantity and integrity was analyzed using Nanodrop (Thermo Fisher) and Bioanalyzer (Agilent). The samples were randomized during library preparation and sequencing was performed on Illumina NovaSeq 6000 at the McGill University Génome Québec Innovation Centre.

\subsubsection{Differential expression}

Read counts per gene were preprocessed (supplementary materials 1.8.1 \& supplementary figure 2). Differential expression (DE) between POL E and SAL E offspring was calculated using the limma-voom pipeline from Bioconductor's limma package (Version 3.40.6) (136). A linear mixed model was used to account for the repeated measurements from each subject to assess $D E$ between groups at each region of interest (ROI) (covarying for sex), as well as global differences between groups (covarying for sex and ROI), correcting for multiple comparisons with FDR (128). A follow up analysis was performed on each sex separately to assess potential sex differences (supplementary materials 1.8.2). Rank rank hypergeometric overlap (RRHO) was used to evaluate the similarity of genome-wide $D E$ in pairs of regions by determining the degree of statistical enrichment using hypergeometric distribution while sliding across all possible thresholds through two ranked lists (https://systems.crump.ucla.edu/rankrank/rankranksimple.php; see supplementary materials 1.8.3).

\subsubsection{Pathway Enrichment, Rank Rank Hypergeometric Overlap, and Disease Gene Analysis}

Pathway enrichment analysis was performed using g:Profiler $(138,139)$ to identify pathways with significantly enriched or overrepresented genes from our gene lists ranked by the - log10P value relative to a background gene list. Pathways include Gene Ontology (GO) terms (biological process, cellular components, molecular function), pathways 
(Reactome, KEGG), networks, regulatory motifs, and disease phenotypes (138). Enrichment significance was assessed using $\mathrm{g}: \mathrm{GOSt}$, applying the hypergeometric distribution. Multiple comparisons correction was performed using g:Profiler's tailor-made algorithm g:SCS (140) (supplementary materials 1.8.4). To measure concordance of differential gene expression patterns between POL E and SAL E across ROIs and between sexes we applied a rank rank hypergeometric overlap test (RRHO) (Cahill et al., 2018); a pathway enrichment analysis was performed on overlap lists (supplementary materials 1.8.3).

Using the Bioconductor GeneOverlap package (https://www.bioconductor.org/packages/release/bioc/html/GeneOverlap.html) in R, dHIP and VHIP DEGs were compared to those identified by human post-mortem studies in the HIP of individuals with schizophrenia (57). Similarly, all our ROls were compared to schizophrenia and ASD pancortical DEGs (56) (supplementary materials 1.8.5).

\section{Disclosures}

None to report

\section{Acknowledgements}

We would like to thank the Ludmer Center of neuroinformatics and mental health (http://ludmercentre.ca/) at McGill University for their support with the transcriptional analysis. Additionally, we would like to thank Gülebru Ayranci, PhD for her help with MRI acquisition, and the Douglas Animal Facility staff for their support with animal care. Finally, we would like to thank Dr's Bruno Giros and Salah El Mestikawy for lending us their centrifuge.

\section{Abbreviations}

MIA: maternal immune activation

GD: gestational day

PND: postnatal day

POL: poly I:C

SAL: saline

E: early (GD9)

L: late (GD17)

OFT: open field test

PPI: prepulse inhibition

SOPT: social preference test

SONT: social novelty test

ASST: attentional set shifting task

FDR: false discovery rate 
PLS: partial least squares

LV: latent variable

ROI: region of interest

ASD: autism spectrum disorder

FGF: fibroblast growth factor

DEG: differentially expressed gene

RRHO: rank rank hypergeometric overlap

QC: quality control

ACC: anterior cingulate cortex

dHIP: dorsal hippocampus

vHIP: ventral hippocampus

\section{References}

1. Knuesel I, Chicha L, Britschgi M, Schobel SA, Bodmer M, Hellings JA, et al. Maternal immune activation and abnormal brain development across CNS disorders. Nat Rev Neurol. 2014 Nov;10(11):643-60.

2. Thomason ME. Development of Brain Networks In Utero: Relevance for Common Neural Disorders. Biol Psychiatry. 2020 Jul 1;88(1):40-50.

3. Selemon LD, Zecevic N. Schizophrenia: a tale of two critical periods for prefrontal cortical development. Transl Psychiatry. 2015 Aug 18;5:e623.

4. Wormser GP, Tolan RW. Infectious Diseases of the Fetus and Newborn Infant, 6th Edition Edited by Jack S. Remington, Jerome O. Klein, Christopher B. Wilson, and Carol J. Baker Philadelphia: Elsevier Saunders, 2006. 1328 pp., illustrated. \$229.00 (cloth) [Internet]. Vol. 42, Clinical Infectious Diseases. 2006. p. 1062-3. Available from: http://dx.doi.org/10.1086/501023

5. Estes ML, McAllister AK. Maternal immune activation: Implications for neuropsychiatric disorders. Science. 2016 Aug 19;353(6301):772-7.

6. Brown AS, Meyer U. Maternal Immune Activation and Neuropsychiatric Illness: A Translational Research Perspective. Am J Psychiatry. 2018 Nov 1;175(11):107383.

7. Meyer U, Feldon J, Yee BK. A review of the fetal brain cytokine imbalance hypothesis of schizophrenia. Schizophr Bull. 2009 Sep;35(5):959-72.

8. Smith SEP, Li J, Garbett K, Mirnics K, Patterson PH. Maternal immune activation alters fetal brain development through interleukin-6. J Neurosci. 2007 Oct 3;27(40):10695-702. 
9. Meyer U, Murray PJ, Urwyler A, Yee BK, Schedlowski M, Feldon J. Adult behavioral and pharmacological dysfunctions following disruption of the fetal brain balance between pro-inflammatory and IL-10-mediated anti-inflammatory signaling [Internet]. Vol. 13, Molecular Psychiatry. 2008. p. 208-21. Available from: http://dx.doi.org/10.1038/sj.mp.4002042

10. Reisinger S, Khan D, Kong E, Berger A, Pollak A, Pollak DD. The Poly(I:C)-induced maternal immune activation model in preclinical neuropsychiatric drug discovery. Pharmacol Ther. 2015 May 1;149:213-26.

11. Gumusoglu SB, Stevens HE. Maternal Inflammation and Neurodevelopmental Programming: A Review of Preclinical Outcomes and Implications for Translational Psychiatry. Biol Psychiatry. 2019 Jan 15;85(2):107-21.

12. Boksa P. Effects of prenatal infection on brain development and behavior: a review of findings from animal models. Brain Behav Immun. 2010 Aug;24(6):881-97.

13. Guma E, Plitman E, Chakravarty MM. The role of maternal immune activation in altering the neurodevelopmental trajectories of offspring: A translational review of neuroimaging studies with implications for autism spectrum disorder and schizophrenia. Neurosci Biobehav Rev [Internet]. 2019; Available from: https://www.sciencedirect.com/science/article/pii/S0149763419302088

14. Haddad FL, Patel SV, Schmid S. Maternal Immune Activation by Poly I:C as a preclinical Model for Neurodevelopmental Disorders: A focus on Autism and Schizophrenia. Neurosci Biobehav Rev. 2020 Jun;113:546-67.

15. Kreitz S, Zambon A, Ronovsky M, Budinsky L, Helbich TH, Sideromenos S, et al. Maternal immune activation during pregnancy impacts on brain structure and function in the adult offspring. Brain Behav Immun. 2020 Jan;83:56-67.

16. Piontkewitz $\mathrm{Y}$, Arad M, Weiner I. Abnormal trajectories of neurodevelopment and behavior following in utero insult in the rat. Biol Psychiatry. 2011 Nov 1;70(9):84251.

17. Meyer U, Nyffeler M, Engler A, Urwyler A, Schedlowski M, Knuesel I, et al. The time of prenatal immune challenge determines the specificity of inflammationmediated brain and behavioral pathology. J Neurosci. 2006 May 3;26(18):4752-62.

18. Dekel N, Gnainsky Y, Granot I, Mor G. Inflammation and implantation. Am J Reprod Immunol. 2010 Jan;63(1):17-21.

19. Mor G, Cardenas I. The immune system in pregnancy: a unique complexity. Am J Reprod Immunol. 2010 Jun;63(6):425-33.

20. Brown AS, Begg MD, Gravenstein S, Schaefer CA, Wyatt RJ, Bresnahan M, et al. 
Serologic evidence of prenatal influenza in the etiology of schizophrenia. Arch Gen Psychiatry. 2004 Aug;61(8):774-80.

21. Atladóttir HÓ, Thorsen P, Østergaard L, Schendel DE, Lemcke S, Abdallah M, et al. Maternal Infection Requiring Hospitalization During Pregnancy and Autism Spectrum Disorders [Internet]. Vol. 40, Journal of Autism and Developmental Disorders. 2010. p. 1423-30. Available from: http://dx.doi.org/10.1007/s10803-0101006-y

22. Ellenbroek B, Youn J. Rodent models in neuroscience research: is it a rat race? Dis Model Mech. 2016 Oct 1;9(10):1079-87.

23. Cope ZA, Powell SB, Young JW. Modeling neurodevelopmental cognitive deficits in tasks with cross-species translational validity: Translating models of neurodevelopmental disorders. Genes Brain Behav. 2016 Jan 14;15(1):27-44.

24. Meyer U, Nyffeler M, Yee BK, Knuesel I, Feldon J. Adult brain and behavioral pathological markers of prenatal immune challenge during early/middle and late fetal development in mice. Brain Behav Immun. 2008 May;22(4):469-86.

25. Li Q, Cheung C, Wei R, Hui ES, Feldon J, Meyer U, et al. Prenatal immune challenge is an environmental risk factor for brain and behavior change relevant to schizophrenia: evidence from MRI in a mouse model. PLoS One. $2009 \mathrm{Jul}$ 24;4(7):e6354.

26. Li Q, Leung YO, Zhou I, Ho LC, Kong W, Basil P, et al. Dietary supplementation with n-3 fatty acids from weaning limits brain biochemistry and behavioural changes elicited by prenatal exposure to maternal inflammation in the mouse model. Transl Psychiatry. 2015 Sep 22;5:e641.

27. Shaw P, Gogtay N, Rapoport J. Childhood psychiatric disorders as anomalies in neurodevelopmental trajectories. Hum Brain Mapp. 2010 Jun;31(6):917-25.

28. Qu LR, Fernandes DJ, Szulc-Lerch KU, Dazai J, Nieman BJ, Turnbull DH, et al. Mouse MRI shows brain areas relatively larger in males emerge before those larger in females. Nat Commun. 2018 Jul 5;9(1):2615.

29. Rapoport JL, Addington AM, Frangou S, Psych MRC. The neurodevelopmental model of schizophrenia: update 2005. Mol Psychiatry. 2005 May;10(5):434-49.

30. Cannon TD, van Erp TGM, Bearden CE, Loewy R, Thompson P, Toga AW, et al. Early and late neurodevelopmental influences in the prodrome to schizophrenia: contributions of genes, environment, and their interactions. Schizophr Bull. 2003;29(4):653-69.

31. Pantelis C, Yücel M, Bora E, Fornito A, Testa R, Brewer WJ, et al. Neurobiological 
markers of illness onset in psychosis and schizophrenia: The search for a moving target. Neuropsychol Rev. 2009 Sep;19(3):385-98.

32. Martins-Filho PR, Tanajura DM, Santos HP Jr, Santos VS. COVID-19 during pregnancy: Potential risk for neurodevelopmental disorders in neonates? Eur $\mathrm{J}$ Obstet Gynecol Reprod Biol. 2020 Jul;250:255-6.

33. Liu H, Wang L-L, Zhao S-J, Kwak-Kim J, Mor G, Liao A-H. Why are pregnant women susceptible to COVID-19? An immunological viewpoint. J Reprod Immunol. 2020 Jun;139:103122.

34. Zimmer A, Youngblood A, Adnane A, Miller BJ, Goldsmith DR. Prenatal exposure to viral infection and neuropsychiatric disorders in offspring: a review of the literature and recommendations for the COVID-19 pandemic. Brain Behav Immun [Internet]. 2020 Nov 2; Available from: http://dx.doi.org/10.1016/j.bbi.2020.10.024

35. Raznahan A, Shaw PW, Lerch JP, Clasen LS, Greenstein D, Berman R, et al. Longitudinal four-dimensional mapping of subcortical anatomy in human development. Proc Natl Acad Sci U S A. 2014 Jan 28;111(4):1592-7.

36. van Erp TGM, Hibar DP, Rasmussen JM, Glahn DC, Pearlson GD, Andreassen $O A$, et al. Subcortical brain volume abnormalities in 2028 individuals with schizophrenia and 2540 healthy controls via the ENIGMA consortium. Mol Psychiatry. 2016 Apr;21(4):547-53.

37. Lieberman JA, Girgis RR, Brucato G, Moore H, Provenzano F, Kegeles L, et al. Hippocampal dysfunction in the pathophysiology of schizophrenia: a selective review and hypothesis for early detection and intervention. Mol Psychiatry. 2018 Aug;23(8):1764-72.

38. Nicolson R, DeVito TJ, Vidal CN, Sui Y, Hayashi KM, Drost DJ, et al. Detection and mapping of hippocampal abnormalities in autism. Psychiatry Res. 2006 Nov 22;148(1):11-21.

39. Bedford SA, Park MTM, Devenyi GA, Tullo S, Germann J, Patel R, et al. Largescale analyses of the relationship between sex, age and intelligence quotient heterogeneity and cortical morphometry in autism spectrum disorder. Mol Psychiatry [Internet]. 2019 Apr 26; Available from: http://dx.doi.org/10.1038/s41380019-0420-6

40. Brisch R, Bernstein H-G, Dobrowolny H, Krell D, Stauch R, Trübner K, et al. A morphometric analysis of the septal nuclei in schizophrenia and affective disorders: reduced neuronal density in the lateral septal nucleus in bipolar disorder. Eur Arch Psychiatry Clin Neurosci. 2011 Feb;261(1):47-58.

41. Li Y, Shen M, Stockton ME, Zhao X. Hippocampal deficits in neurodevelopmental 
disorders. Neurobiol Learn Mem. 2019 Nov;165:106945.

42. Cheung C, Yu K, Fung G, Leung M, Wong C, Li Q, et al. Autistic disorders and schizophrenia: related or remote? An anatomical likelihood estimation. PLoS One. 2010 Aug 18;5(8):e12233.

43. Radulescu E, Ganeshan B, Shergill SS, Medford N, Chatwin C, Young RCD, et al. Grey-matter texture abnormalities and reduced hippocampal volume are distinguishing features of schizophrenia. Psychiatry Res. 2014 Sep 30;223(3):17986.

44. Fujiwara H, Hirao K, Namiki C, Yamada M, Shimizu M, Fukuyama H, et al. Anterior cingulate pathology and social cognition in schizophrenia: a study of gray matter, white matter and sulcal morphometry. Neuroimage. 2007 Jul 15;36(4):1236-45.

45. Calabrese DR, Wang L, Harms MP, Ratnanather JT, Barch DM, Cloninger CR, et al. Cingulate gyrus neuroanatomy in schizophrenia subjects and their nonpsychotic siblings. Schizophr Res. 2008 Sep;104(1-3):61-70.

46. Simms ML, Kemper TL, Timbie CM, Bauman ML, Blatt GJ. The anterior cingulate cortex in autism: heterogeneity of qualitative and quantitative cytoarchitectonic features suggests possible subgroups. Acta Neuropathol. 2009 Nov;118(5):673-84.

47. Schumann CM, Bloss CS, Barnes CC, Wideman GM, Carper RA, Akshoomoff N, et al. Longitudinal magnetic resonance imaging study of cortical development through early childhood in autism. J Neurosci. 2010 Mar 24;30(12):4419-27.

48. Starace D, Galli R, Paone A, De Cesaris P, Filippini A, Ziparo E, et al. Toll-like receptor 3 activation induces antiviral immune responses in mouse sertoli cells. Biol Reprod. 2008 Oct;79(4):766-75.

49. Balasubramanian $R$, Zhang $X$. Mechanisms of FGF gradient formation during embryogenesis. Semin Cell Dev Biol. 2016 May;53:94-100.

50. Roth KA, D'Sa C. Apoptosis and brain development. Ment Retard Dev Disabil Res Rev. 2001;7(4):261-6.

51. Bergdolt L, Dunaevsky A. Brain changes in a maternal immune activation model of neurodevelopmental brain disorders. Prog Neurobiol. 2019 Apr;175:1-19.

52. Morera $D$, MacKenzie SA. Is there a direct role for erythrocytes in the immune response? Vet Res. 2011 Jul 29;42:89.

53. Ferreiro DU, Komives EA. Molecular mechanisms of system control of NF-kappaB signaling by IkappaBalpha. Biochemistry. 2010 Mar 2;49(8):1560-7.

54. Choi GB, Yim YS, Wong H, Kim S, Kim H, Kim SV, et al. The maternal interleukin- 
17a pathway in mice promotes autism-like phenotypes in offspring [Internet]. Vol. 351, Science. 2016. p. 933-9. Available from:

http://dx.doi.org/10.1126/science.aad0314

55. Gabay C, Lamacchia C, Palmer G. IL-1 pathways in inflammation and human diseases. Nat Rev Rheumatol. 2010 Apr;6(4):232-41.

56. Gandal MJ, Haney JR, Parikshak NN, Leppa V, Ramaswami G, Hartl C, et al. Shared molecular neuropathology across major psychiatric disorders parallels polygenic overlap. Science. 2018 Feb 9;359(6376):693-7.

57. Lanz TA, Reinhart V, Sheehan MJ, Rizzo SJS, Bove SE, James LC, et al. Postmortem transcriptional profiling reveals widespread increase in inflammation in schizophrenia: a comparison of prefrontal cortex, striatum, and hippocampus among matched tetrads of controls with subjects diagnosed with schizophrenia, bipolar or major depressive disorder. Transl Psychiatry. 2019 May 23;9(1):151.

58. Piontkewitz $\mathrm{Y}$, Arad M, Weiner I. Tracing the development of psychosis and its prevention: what can be learned from animal models. Neuropharmacology. 2012 Mar;62(3):1273-89.

59. Wolff JJ, Jacob S, Elison JT. The journey to autism: Insights from neuroimaging studies of infants and toddlers. Dev Psychopathol. 2018 May;30(2):479-95.

60. Lerch JP, Gazdzinski L, Germann J, Sled JG, Henkelman RM, Nieman BJ. Wanted dead or alive? The tradeoff between in-vivo versus ex-vivo MR brain imaging in the mouse. Front Neuroinform. 2012 Mar 23;6:6.

61. Mills KL, Tamnes CK. Methods and considerations for longitudinal structural brain imaging analysis across development. Dev Cogn Neurosci. 2014 Jul;9:172-90.

62. Gur RE, Keshavan MS, Lawrie SM. Deconstructing psychosis with human brain imaging. Schizophr Bull. 2007 Jul;33(4):921-31.

63. Nieman BJ, Bishop J, Dazai J, Bock NA, Lerch JP, Feintuch A, et al. MR technology for biological studies in mice. NMR Biomed. 2007 May;20(3):291-303.

64. Gallino D, Devenyi GA, Germann J, Guma E, Anastassiadis C, Chakravarty MM. Longitudinal assessment of the neuroanatomical consequences of deep brain stimulation: Application of fornical DBS in an Alzheimer's mouse model. Brain Res. 2019 Jul 15;1715:213-23.

65. Rollins CPE, Gallino D, Kong V, Ayranci G, Devenyi GA, Germann J, et al. Contributions of a high-fat diet to Alzheimer's disease-related decline: A longitudinal behavioural and structural neuroimaging study in mouse models. Neurolmage: Clinical. 2019 Jan 1;21:101606. 
66. Guma E, Rocchetti J, Devenyi GA, Tanti A, Mathieu A, Lerch JP, et al. Regional brain volume changes following chronic antipsychotic administration are mediated by the dopamine D2 receptor. Neuroimage. 2018 Apr 26;176:226-38.

67. Guma E, Rocchetti J, Devenyi GA, Tanti A, Mathieu AP, Lerch JP, et al. Role of D3 dopamine receptors in modulating neuroanatomical changes in response to antipsychotic administration. Sci Rep. 2019 May 24;9(1):7850.

68. Kong V, Devenyi GA, Gallino D, Ayranci G, Germann J, Rollins C, et al. Early-in-life neuroanatomical and behavioural trajectories in a triple transgenic model of Alzheimer's disease. Brain Struct Funct [Internet]. 2018 Jun 13; Available from: http://dx.doi.org/10.1007/s00429-018-1691-4

69. Szulc KU, Lerch JP, Nieman BJ, Bartelle BB, Friedel M, Suero-Abreu GA, et al. 4D MEMRI atlas of neonatal FVB/N mouse brain development. Neuroimage. 2015 Sep;118:49-62.

70. Varghese M, Keshav N, Jacot-Descombes S, Warda T, Wicinski B, Dickstein DL, et al. Autism spectrum disorder: neuropathology and animal models. Acta Neuropathol. 2017 Oct;134(4):537-66.

71. Baron-Cohen S, Ring HA, Bullmore ET, Wheelwright S, Ashwin C, Williams SC. The amygdala theory of autism. Neurosci Biobehav Rev. 2000 May;24(3):355-64.

72. Koropouli E, Melanitis N, Dimitriou VI, Grigoriou A, Karavasilis E, Nikita KS, et al. New-Onset Psychosis Associated With a Lesion Localized in the Rostral Tectum: Insights Into Pathway-Specific Connectivity Disrupted in Psychosis. Schizophr Bull [Internet]. 2020 Feb 26; Available from: http://dx.doi.org/10.1093/schbul/sbaa018

73. George DT, Ameli R, Koob GF. Periaqueductal Gray Sheds Light on Dark Areas of Psychopathology. Trends Neurosci. 2019 May;42(5):349-60.

74. Freeman SM, Palumbo MC, Lawrence RH, Smith AL, Goodman MM, Bales KL. Effect of age and autism spectrum disorder on oxytocin receptor density in the human basal forebrain and midbrain. Transl Psychiatry. 2018 Dec 4;8(1):257.

75. Shin Yim Y, Park A, Berrios J, Lafourcade M, Pascual LM, Soares N, et al. Reversing behavioural abnormalities in mice exposed to maternal inflammation. Nature. 2017 Sep 28;549(7673):482-7.

76. Balasco L, Provenzano G, Bozzi Y. Sensory Abnormalities in Autism Spectrum Disorders: A Focus on the Tactile Domain, From Genetic Mouse Models to the Clinic. Front Psychiatry. 2019;10:1016.

77. Teale P, Pasko B, Collins D, Rojas D, Reite M. Somatosensory timing deficits in schizophrenia. Psychiatry Res. 2013 Apr 30;212(1):73-8. 
78. Butler T, Weisholtz D, Isenberg N, Harding E, Epstein J, Stern E, et al. Neuroimaging of frontal-limbic dysfunction in schizophrenia and epilepsy-related psychosis: toward a convergent neurobiology. Epilepsy Behav. 2012 Feb;23(2):113-22.

79. Wegiel J, Flory M, Kuchna I, Nowicki K, Ma SY, Imaki H, et al. Brain-region-specific alterations of the trajectories of neuronal volume growth throughout the lifespan in autism. Acta Neuropathol Commun. 2014 Mar 10;2:28.

80. Hazlett HC, Poe MD, Gerig G, Styner M, Chappell C, Smith RG, et al. Early brain overgrowth in autism associated with an increase in cortical surface area before age 2 years. Arch Gen Psychiatry. 2011 May;68(5):467-76.

81. Donovan APA, Basson MA. The neuroanatomy of autism--a developmental perspective. J Anat. 2017;230(1):4-15.

82. Kentner AC, Bilbo SD, Brown AS, Hsiao EY, McAllister AK, Meyer U, et al. Maternal immune activation: reporting guidelines to improve the rigor, reproducibility, and transparency of the model. Neuropsychopharmacology. 2019 Jan;44(2):245-58.

83. Richetto J, Chesters R, Cattaneo A, Labouesse MA, Gutierrez AMC, Wood TC, et al. Genome-Wide Transcriptional Profiling and Structural Magnetic Resonance Imaging in the Maternal Immune Activation Model of Neurodevelopmental Disorders. Cereb Cortex. 2017 Jun 1;27(6):3397-413.

84. Crum WR, Sawiak SJ, Chege W, Cooper JD, Williams SCR, Vernon AC. Evolution of structural abnormalities in the rat brain following in utero exposure to maternal immune activation: A longitudinal in vivo MRI study. Brain Behav Immun. 2017 Jul;63:50-9.

85. Bitanihirwe BKY, Peleg-Raibstein D, Mouttet F, Feldon J, Meyer U. Late Prenatal Immune Activation in Mice Leads to Behavioral and Neurochemical Abnormalities Relevant to the Negative Symptoms of Schizophrenia [Internet]. Vol. 35, Neuropsychopharmacology. 2010. p. 2462-78. Available from: http://dx.doi.org/10.1038/npp.2010.129

86. Meyer U, Nyffeler M, Schwendener S, Knuesel I, Yee BK, Feldon J. Relative prenatal and postnatal maternal contributions to schizophrenia-related neurochemical dysfunction after in utero immune challenge. Neuropsychopharmacology. 2008 Jan;33(2):441-56.

87. Giovanoli S, Engler H, Engler A, Richetto J, Voget M, Willi R, et al. Stress in Puberty Unmasks Latent Neuropathological Consequences of Prenatal Immune Activation in Mice [Internet]. Vol. 339, Science. 2013. p. 1095-9. Available from: http://dx.doi.org/10.1126/science.1228261 
88. Conway F, Brown AS. Maternal Immune Activation and Related Factors in the Risk of Offspring Psychiatric Disorders. Front Psychiatry. 2019 Jun 28;10:430.

89. Semple BD, Blomgren K, Gimlin K, Ferriero DM, Noble-Haeusslein LJ. Brain development in rodents and humans: Identifying benchmarks of maturation and vulnerability to injury across species [Internet]. Vols. 106-107, Progress in Neurobiology. 2013. p. 1-16. Available from: http://dx.doi.org/10.1016/j.pneurobio.2013.04.001

90. Clancy B, Finlay BL, Darlington RB, Anand KJS. Extrapolating brain development from experimental species to humans. Neurotoxicology. 2007 Sep;28(5):931-7.

91. Clancy B, Darlington RB, Finlay BL. Translating developmental time across mammalian species. Neuroscience. 2001;105(1):7-17.

92. Le Belle JE, Sperry J, Ngo A, Ghochani Y, Laks DR, López-Aranda M, et al. Maternal inflammation contributes to brain overgrowth and autism-associated behaviors through altered redox signaling in stem and progenitor cells. Stem Cell Reports. 2014 Nov 11;3(5):725-34.

93. Tronnes AA, Koschnitzky J, Daza R, Hitti J, Ramirez JM, Hevner R. Effects of Lipopolysaccharide and Progesterone Exposures on Embryonic Cerebral Cortex Development in Mice. Reprod Sci. 2016 Jun;23(6):771-8.

94. Manitz MP, Plümper J, Demir S, Ahrens M, Eßlinger M, Wachholz S, et al. Flow cytometric characterization of microglia in the offspring of Polyl:C treated mice [Internet]. Vol. 1636, Brain Research. 2016. p. 172-82. Available from: http://dx.doi.org/10.1016/j.brainres.2016.02.004

95. Pendyala G, Chou S, Jung Y, Coiro P, Spartz E, Padmashri R, et al. Maternal Immune Activation Causes Behavioral Impairments and Altered Cerebellar Cytokine and Synaptic Protein Expression. Neuropsychopharmacology. 2017 Jun;42(7):1435-46.

96. Cui K, Ashdown H, Luheshi GN, Boksa P. Effects of prenatal immune activation on hippocampal neurogenesis in the rat. Schizophr Res. 2009 Sep;113(2-3):288-97.

97. Schnack $\mathrm{H}$. Assessing reproducibility in association studies. Elife [Internet]. 2019 Apr 25;8. Available from: http://dx.doi.org/10.7554/eLife.46757

98. Petersen SE, Sporns O. Brain Networks and Cognitive Architectures. Neuron. 2015 Oct 7;88(1):207-19.

99. Di Martino A, Fair DA, Kelly C, Satterthwaite TD, Castellanos FX, Thomason ME, et al. Unraveling the miswired connectome: a developmental perspective. Neuron. 2014 Sep 17;83(6):1335-53. 
100. Xia CH, Ma Z, Ciric R, Gu S, Betzel RF, Kaczkurkin AN, et al. Linked dimensions of psychopathology and connectivity in functional brain networks. Nat Commun. 2018 Aug 1;9(1):3003.

101. Uddin LQ, Karlsgodt KH. Future Directions for Examination of Brain Networks in Neurodevelopmental Disorders. J Clin Child Adolesc Psychol. 2018 May;47(3):483-97.

102. Ball G, Seidlitz J, Beare R, Seal ML. Cortical remodelling in childhood is associated with genes enriched for neurodevelopmental disorders. Neuroimage. 2020 Jul 15;215:116803.

103. Shafiei G, Markello RD, Makowski C, Talpalaru A, Kirschner M, Devenyi GA, et al. Spatial Patterning of Tissue Volume Loss in Schizophrenia Reflects Brain Network Architecture. Biol Psychiatry. 2020 Apr 15;87(8):727-35.

104. Kirschner M, Shafiei G, Markello RD, Makowski C, Talpalaru A, Hodzic-Santor B, et al. Latent Clinical-Anatomical Dimensions of Schizophrenia. Schizophr Bull [Internet]. 2020 Aug 3; Available from: http://dx.doi.org/10.1093/schbul/sbaa097

105. Ratnayake U, Quinn T, Walker DW, Dickinson H. Cytokines and the neurodevelopmental basis of mental illness. Front Neurosci. 2013 Oct 17;7:180.

106. Rudolph MD, Graham AM, Feczko E, Miranda-Dominguez O, Rasmussen JM, Nardos R, et al. Maternal IL-6 during pregnancy can be estimated from newborn brain connectivity and predicts future working memory in offspring. Nat Neurosci. 2018 May;21(5):765-72.

107. Diez Del Corral R, Morales AV. The Multiple Roles of FGF Signaling in the Developing Spinal Cord. Front Cell Dev Biol. 2017 Jun 2;5:58.

108. Terwisscha van Scheltinga AF, Bakker SC, Kahn RS. Fibroblast growth factors in schizophrenia. Schizophr Bull. 2010 Nov;36(6):1157-66.

109. Reuss B, von Bohlen und Halbach O. Fibroblast growth factors and their receptors in the central nervous system [Internet]. Vol. 313, Cell and Tissue Research. 2003. p. 139-57. Available from: http://dx.doi.org/10.1007/s00441-0030756-7

110. Guillemot F, Zimmer C. From cradle to grave: the multiple roles of fibroblast growth factors in neural development. Neuron. 2011 Aug 25;71(4):574-88.

111. Matsumoto N, Shinmyo Y, Ichikawa Y, Kawasaki H. Gyrification of the cerebral cortex requires FGF signaling in the mammalian brain. Elife [Internet]. 2017 Nov 14;6. Available from: http://dx.doi.org/10.7554/eLife.29285 
112. Nandi S, Alviña K, Lituma PJ, Castillo PE, Hébert JM. Neurotrophin and FGF Signaling Adapter Proteins, FRS2 and FRS3, Regulate Dentate Granule Cell Maturation and Excitatory Synaptogenesis. Neuroscience. 2018 Jan 15;369:192201.

113. Dabrowski A, Terauchi A, Strong C, Umemori H. Distinct sets of FGF receptors sculpt excitatory and inhibitory synaptogenesis. Development. 2015 May 15;142(10):1818-30.

114. Neugebauer JM, Yost HJ. FGF signaling is required for brain left-right asymmetry and brain midline formation. Dev Biol. 2014 Feb 1;386(1):123-34.

115. Duman RS, Monteggia LM. A Neurotrophic Model for Stress-Related Mood Disorders [Internet]. Vol. 59, Biological Psychiatry. 2006. p. 1116-27. Available from: http://dx.doi.org/10.1016/j.biopsych.2006.02.013

116. Perez JA, Clinton SM, Turner CA, Watson SJ, Akil H. A new role for FGF2 as an endogenous inhibitor of anxiety. J Neurosci. 2009 May 13;29(19):6379-87.

117. Rubenstein JLR. Three hypotheses for developmental defects that may underlie some forms of autism spectrum disorder. Curr Opin Neurol. 2010 Apr;23(2):11823.

118. Vaccarino FM, Grigorenko EL, Smith KM, Stevens HE. Regulation of cerebral cortical size and neuron number by fibroblast growth factors: implications for autism. J Autism Dev Disord. 2009 Mar;39(3):511-20.

119. O'Donovan MC, Norton N, Williams H, Peirce T, Moskvina V, Nikolov I, et al. Analysis of 10 independent samples provides evidence for association between schizophrenia and a SNP flanking fibroblast growth factor receptor 2. Mol Psychiatry. 2009 Jan;14(1):30-6.

120. Jungerius BJ, Hoogendoorn MLC, Bakker SC, Van't Slot R, Bardoel AF, Ophoff RA, et al. An association screen of myelin-related genes implicates the chromosome 22q11 PIK4CA gene in schizophrenia. Mol Psychiatry. 2008 Nov;13(11):1060-8.

121. Patel S, Dale RC, Rose D, Heath B, Nordahl CW, Rogers S, et al. Maternal immune conditions are increased in males with autism spectrum disorders and are associated with behavioural and emotional but not cognitive co-morbidity. Transl Psychiatry. 2020 Aug 14;10(1):286.

122. Buka SL, Tsuang MT, Torrey EF, Klebanoff MA, Bernstein D, Yolken RH. Maternal infections and subsequent psychosis among offspring. Arch Gen Psychiatry. 2001 Nov;58(11):1032-7. 
123. Cossío LF de, de Cossío LF, Guzmán A, van der Veldt S, Luheshi GN. Prenatal infection leads to ASD-like behavior and altered synaptic pruning in the mouse offspring [Internet]. Vol. 63, Brain, Behavior, and Immunity. 2017. p. 88-98.

Available from: http://dx.doi.org/10.1016/j.bbi.2016.09.028

124. Vousden DA, Cox E, Allemang-Grand R, Laliberté C, Qiu LR, Lindenmaier Z, et al. Continuous manganese delivery via osmotic pumps for manganese-enhanced mouse MRI does not impair spatial learning but leads to skin ulceration. Neuroimage. 2018 Jun;173:411-20.

125. Friedel M, van Eede MC, Pipitone J, Chakravarty MM, Lerch JP. Pydpiper: a flexible toolkit for constructing novel registration pipelines. Front Neuroinform. 2014 Jul 30;8:67.

126. van Eede MC, Scholz J, Chakravarty MM, Henkelman RM, Lerch JP. Mapping registration sensitivity in MR mouse brain images. Neuroimage. $2013 \mathrm{Nov}$ 15;82:226-36.

127. Chung MK, Worsley KJ, Paus T, Cherif C, Collins DL, Giedd JN, et al. A unified statistical approach to deformation-based morphometry. Neuroimage. 2001 Sep;14(3):595-606.

128. Benjamini $\mathrm{Y}$, Hochberg Y. Controlling the False Discovery Rate: A Practical and Powerful Approach to Multiple Testing. J R Stat Soc Series B Stat Methodol. 1995;57(1):289-300.

129. Deacon RMJ. Digging and marble burying in mice: simple methods for in vivo identification of biological impacts. Nat Protoc. 2006;1(1):122-4.

130. Colacicco G, Welzl H, Lipp H-P, Würbel H. Attentional set-shifting in mice: modification of a rat paradigm, and evidence for strain-dependent variation. Behav Brain Res. 2002 Apr 15;132(1):95-102.

131. Zeighami Y, Fereshtehnejad S-M, Dadar M, Collins DL, Postuma RB, Mišić B, et al. A clinical-anatomical signature of Parkinson's disease identified with partial least squares and magnetic resonance imaging. Neuroimage. 2019 Apr 15;190:69-78.

132. Mclntosh AR, Mišić B. Multivariate statistical analyses for neuroimaging data. Annu Rev Psychol. 2013;64:499-525.

133. McIntosh AR, Lobaugh NJ. Partial least squares analysis of neuroimaging data: applications and advances. Neuroimage. 2004;23 Suppl 1:S250-63.

134. Eckart $\mathrm{C}$, Young $\mathrm{G}$. The approximation of one matrix by another of lower rank. Psychometrika. 1936 Sep 1;1(3):211-8. 
135. Patel R, Steele CJ, Chen AGX, Patel S, Devenyi GA, Germann J, et al. Investigating microstructural variation in the human hippocampus using nonnegative matrix factorization. Neuroimage. 2020 Feb 15;207:116348.

136. Ritchie ME, Phipson B, Wu D, Hu Y, Law CW, Shi W, et al. limma powers differential expression analyses for RNA-sequencing and microarray studies. Nucleic Acids Res. 2015 Apr 20;43(7):e47.

137. Plaisier SB, Taschereau R, Wong JA, Graeber TG. Rank-rank hypergeometric overlap: identification of statistically significant overlap between gene-expression signatures. Nucleic Acids Res. 2010 Jul 21;38(17):e169-e169.

138. Reimand J, Isserlin R, Voisin V, Kucera M, Tannus-Lopes C, Rostamianfar A, et al. Pathway enrichment analysis and visualization of omics data using g:Profiler, GSEA, Cytoscape and EnrichmentMap [Internet]. Vol. 14, Nature Protocols. 2019. p. 482-517. Available from: http://dx.doi.org/10.1038/s41596-018-0103-9

139. Reimand J, Kull M, Peterson H, Hansen J, Vilo J. g:Profiler-a web-based toolset for functional profiling of gene lists from large-scale experiments [Internet]. Vol. 35, Nucleic Acids Research. 2007. p. W193-200. Available from: http://dx.doi.org/10.1093/nar/gkm226

140. Reimand J, Arak T, Adler P, Kolberg L, Reisberg S, Peterson H, et al. g:Profiler-a web server for functional interpretation of gene lists (2016 update). Nucleic Acids Res. 2016 Jul 8;44(W1):W83-9. 